\title{
Electroweak corrections in the 2HDM for neutral scalar Higgs-boson production through gluon fusion
}

\author{
Laura Jenniches, ${ }^{a}$ Christian Sturm ${ }^{a}$ and Sandro Uccirati ${ }^{b}$ \\ ${ }^{a}$ Universität Würzburg, Institut für Theoretische Physik und Astrophysik, \\ Lehrstuhl für Theoretische Physik II, Campus Hubland Nord, \\ Emil-Hilb-Weg 22, D-97074 Würzburg, Germany \\ ${ }^{b}$ Università di Torino and INFN, \\ 10125 Torino, Italy \\ E-mail: Laura.Jenniches@physik.uni-wuerzburg.de, \\ Christian.Sturm@physik.uni-wuerzburg.de, uccirati@to.infn.it
}

ABSTRACT: We have computed the two-loop, electroweak corrections to the production of a light and a heavy neutral, scalar Higgs-boson through the important gluon fusion process in the Two-Higgs-Doublet Model. We provide our results in various renormalization schemes for different scenarios and benchmark points, which will be valuable for experimental studies at the LHC. We describe the technicalities of our two-loop calculation and augment it by a phenomenological discussion. Our results are also applicable to the gluonic neutral, scalar Higgs-boson decays.

Keywords: Beyond Standard Model, Higgs Physics

ARXIV EPRINT: 1805.05869 


\section{Contents}

1 Introduction 1

2 The model 2

3 Renormalization of the mixing angles $\alpha$ and $\beta \quad 5$

4 Calculation $\quad 9$

$\begin{array}{ll}4.1 & \text { Analytical and numerical tests } \\ \end{array}$

5 Results and discussion $\quad 13$

6 Summary and conclusion $\quad 23$

A Scale dependence of the percentage correction in the $\overline{\mathrm{MS}}$ scheme without running of $c_{\alpha \beta}$ and $t_{\beta}$

B Behaviour of the coupling constants $\lambda_{i}$

C $\overline{\mathrm{MS}}$ results in the $M^{*}$ benchmark scenarios

\section{Introduction}

The Higgs boson, which has been discovered at the Large Hadron Collider (LHC) $[1,2]$ with a mass of $M_{h}=125.09 \pm 0.21 \pm 0.11 \mathrm{GeV}$ [3], could be part of an extended Higgs sector. One of the simplest extensions of the Standard Model (SM) is the Two-HiggsDoublet Model (2HDM), where an extra scalar doublet is added. Such an extension was introduced in ref. [4] to provide an additional source of CP-violation, which may contribute to explain the observed matter-anti-matter asymmetry of the Universe. Special choices of the parameters, for example in the Inert Model, can also provide a dark matter candidate [5, 6]. Thus, the $2 \mathrm{HDM}$ can assist to address problems which are not solved within the SM. Phenomenological studies of the 2HDM have been performed by the ATLAS [7-14] and CMS [15-21] collaborations. Several benchmark scenarios for the new parameters, which arise due to the addition of a second scalar doublet, have been collected by the LHC Higgs Cross Section Working Group in ref. [22].

Extensions of the SM can strongly modify Higgs-boson production and decay processes, which allows to perform exclusion studies for the new parameters of such model extensions. This requires precise theoretical predictions. For example, the addition of a sequential fourth generation of heavy fermions increases the leading order (LO) cross section of the Higgs-boson production process through gluon fusion already by about a factor of nine [23]. The next-to-leading order (NLO) electroweak corrections in this model have been computed in refs. [24, 25], which have helped to exclude this model at the LHC. 
In the CP-conserving 2HDM, there are two neutral, scalar Higgs bosons $H_{1}$ and $H_{\mathrm{h}}$. One of them is considered to be the SM-like Higgs boson, which has already been discovered at the LHC. In addition, there are two charged Higgs bosons $H^{ \pm}$as well as a pseudoscalar Higgs boson $H_{\mathrm{a}}$. A possible set of new free parameters of the extended Higgs sector are the masses of the new Higgs bosons, $M_{H_{\mathrm{h}}}, M_{H^{ \pm}}, M_{H_{\mathrm{a}}}$ as well as the soft $Z_{2}$-breaking scale $M_{\mathrm{sb}}$ and two mixing angles $\alpha$ and $\beta$. The mass of the light Higgs boson is here fixed to $M_{H_{1}} \equiv M_{h}$.

Various important Higgs-boson production and decay processes have already been studied at NLO in the 2HDM. For example, NLO electroweak corrections to Higgs-boson production in Higgs strahlung and through vector-boson fusion have been determined in the $2 \mathrm{HDM}$ in refs. [26, 27]. The NLO electroweak corrections to the decay of the light Higgs boson of the $2 \mathrm{HDM}$ into four fermions have been computed in refs. [28, 29]. The dominant Higgs-boson production mechanism at the LHC proceeds via gluon fusion. Its precise theoretical knowledge is thus a mandatory task. In the SM, the complete NLO electroweak corrections for the most recent value of the top-quark mass $m_{t}=173.1 \mathrm{GeV}[3]$ amount to $5.1 \%[30,31]$. One can thus expect that the electroweak corrections are also sizable in extensions of the SM. In this work, we will contribute to the effort of studying the 2HDM by computing the two-loop electroweak corrections to the production of neutral, scalar Higgs bosons in gluon fusion. On the one hand, it is important to know the NLO electroweak corrections in the $2 \mathrm{HDM}$ to Higgs-boson production in gluon fusion for the already discovered Higgs boson. First results in the special case of the alignment limit $(\cos (\alpha-\beta)=0)$ have already been presented in ref. [26]. In addition to the alignment limit, we consider the more complicated, general case of $\cos (\alpha-\beta) \neq 0$ in this work. On the other hand, it is important to know the NLO electroweak corrections for the production of the heavy, neutral, scalar Higgs boson $H_{\mathrm{h}}$ of the $2 \mathrm{HDM}$, which we present too. We also discuss the technicalities and obstacles which need to be overcome in order to accomplish this calculation. The results of our computations will be provided in different renormalization schemes for the mixing angles $\alpha$ and $\beta$.

The structure of this paper is as follows: in section 2, we introduce the model in which we perform our calculation. Section 3 describes the different renormalization schemes which we use for the mixing angles. In section 4 , we discuss the computational techniques which have been used in order to accomplish this aim; and in section 5 we provide the numerical results. Finally, we close with our summary and conclusion in section 6 . In the appendices, we provide supplementary information on the scale dependence arising from the $\overline{\mathrm{MS}}$ renormalization of the mixing angles $\alpha$ and $\beta$ as well as on the perturbative behaviour of the coupling constants of the Higgs potential.

\section{The model}

We discuss the 2HDM extension of the SM, which has two complex, scalar doublet fields $\Phi_{i}, i=1,2$. In the generic basis, they are parametrized through

$$
\Phi_{i}=\left(\begin{array}{c}
\phi_{i}^{+} \\
\frac{1}{\sqrt{2}}\left(v_{i}+\rho_{i}+\mathrm{i} \eta_{i}\right)
\end{array}\right),
$$


where the $v_{i}$ are the vacuum expectation values (vev). We consider the $2 \mathrm{HDM}$ with a discrete $Z_{2}$ symmetry under the transformation $\Phi_{1} \rightarrow-\Phi_{1}, \Phi_{2} \rightarrow \Phi_{2}$ of the two Higgs doublets. This $Z_{2}$ symmetry is important, since it has also implications on the Yukawa sector of the 2HDM where it suppresses tree-level flavour-changing neutral currents (FCNC), which are experimentally unobserved. Before coming to the Yukawa sector, let us complete the discussion of the Higgs potential. The $Z_{2}$ symmetry requirement also reduces the number of terms in the potential. However, we allow for a soft-breaking term [32], which does not induce the FCNC problem. With these constraints, the Higgs potential has the following form

$$
\begin{aligned}
V\left(\Phi_{1}, \Phi_{2}\right)= & m_{1}^{2} \Phi_{1}^{\dagger} \Phi_{1}+m_{2}^{2} \Phi_{2}^{\dagger} \Phi_{2}-m_{12}^{2}\left(\Phi_{1}^{\dagger} \Phi_{2}+\Phi_{2}^{\dagger} \Phi_{1}\right) \\
& +\frac{\lambda_{1}}{2}\left(\Phi_{1}^{\dagger} \Phi_{1}\right)^{2}+\frac{\lambda_{2}}{2}\left(\Phi_{2}^{\dagger} \Phi_{2}\right)^{2}+\lambda_{3}\left(\Phi_{1}^{\dagger} \Phi_{1}\right)\left(\Phi_{2}^{\dagger} \Phi_{2}\right) \\
& +\lambda_{4}\left(\Phi_{1}^{\dagger} \Phi_{2}\right)\left(\Phi_{2}^{\dagger} \Phi_{1}\right)+\frac{\lambda_{5}}{2}\left[\left(\Phi_{1}^{\dagger} \Phi_{2}\right)^{2}+\left(\Phi_{2}^{\dagger} \Phi_{1}\right)^{2}\right] .
\end{aligned}
$$

The five couplings $\lambda_{1}, \ldots, \lambda_{5}$ and the soft-breaking parameter $m_{12}$ are taken to be real as well as the two masses $m_{1}$ and $m_{2}$.

For physical applications, we work in the physical basis, where the sector of the potential that is quadratic in the scalar fields is diagonalized. This leads to the introduction of the mass eigenstates through a change of basis

$$
\left(\begin{array}{c}
\rho_{1} \\
\rho_{2}
\end{array}\right)=R(\alpha)\left(\begin{array}{c}
H_{h} \\
H_{l}
\end{array}\right), \quad\left(\begin{array}{c}
\phi_{1}^{ \pm} \\
\phi_{2}^{ \pm}
\end{array}\right)=R(\beta)\left(\begin{array}{c}
G^{ \pm} \\
H^{ \pm}
\end{array}\right), \quad\left(\begin{array}{l}
\eta_{1} \\
\eta_{2}
\end{array}\right)=R(\beta)\left(\begin{array}{c}
G_{0} \\
H_{\mathrm{a}}
\end{array}\right) .
$$

Here, the symbols $H_{\mathrm{l}}, H_{\mathrm{h}}, H^{ \pm} \equiv H_{\mathrm{c}}$ and $H_{\mathrm{a}}$ are the fields of the physical light, heavy, charged and pseudoscalar Higgs bosons, which receive the masses $M_{H_{1}}, M_{H_{\mathrm{h}}}, M_{H_{\mathrm{c}}}$ and $M_{H_{\mathrm{a}}}$, while $G_{0}$ and $G^{ \pm}$are the neutral and charged would-be Goldstone bosons.

The rotation matrix that performs the diagonalization reads

$$
R(\gamma)=\left(\begin{array}{cc}
\cos \gamma & -\sin \gamma \\
\sin \gamma & \cos \gamma
\end{array}\right), \quad \text { with } \quad \gamma=\alpha \text { or } \beta
$$

The five couplings in the potential can then be expressed in terms of the Higgs-boson masses and the mixing angles. The explicit formulae are given in eqs. (B.1)-(B.5) of appendix B.

Finally, we also introduce the Higgs basis [33] in which only the neutral component of one of the two Higgs doublets, say the first one, acquires a vev. This is achieved by the linear combination

$$
\left(\begin{array}{c}
\Phi_{a} \\
\Phi_{b}
\end{array}\right)=R^{-1}(\beta)\left(\begin{array}{l}
\Phi_{1} \\
\Phi_{2}
\end{array}\right)
$$

Expressing the fields $\Phi_{a, b}$ in terms of the fields in the physical basis leads to

$$
\Phi_{a}=\left(\begin{array}{c}
G^{+} \\
\frac{1}{\sqrt{2}}\left(v+\widehat{H}+\mathrm{i} G_{0}\right)
\end{array}\right), \quad \Phi_{b}=\left(\begin{array}{c}
H^{+} \\
\frac{1}{\sqrt{2}}\left(\widetilde{H}+\mathrm{i} H_{\mathrm{a}}\right)
\end{array}\right)
$$


where we defined the neutral Higgs-boson fields through the following linear combinations

$$
\left(\begin{array}{l}
\widetilde{H} \\
\widehat{H}
\end{array}\right)=R^{-1}(\alpha-\beta)\left(\begin{array}{l}
H_{1} \\
H_{\mathrm{h}}
\end{array}\right) .
$$

It becomes apparent that only the neutral component of $\Phi_{a}$ acquires a vev, while in $\Phi_{b}$ it does not. We introduce the abbreviations $c_{\alpha \beta}=\cos (\alpha-\beta)$ and $s_{\alpha \beta}=\sin (\alpha-\beta)$ as well as the vev $v=\left(v_{1}^{2}+v_{2}^{2}\right)^{1 / 2}$. The choice of phases in the definition of the two doublets $\Phi_{i}$ in eq. (2.1) leads to $s_{\alpha \beta}=-\sqrt{1-c_{\alpha \beta}^{2}}$. An additional property of the Higgs basis is that the Goldstone bosons are all contained in one doublet, $\Phi_{a}$. Note, that the Higgs basis was particularly valuable in ref. [26] in order to show that an $\overline{\mathrm{MS}}$ renormalization of the mixing angle $\beta$ can become gauge dependent when using popular tadpole renormalization schemes. In the case of the alignment limit

$$
c_{\alpha \beta} \rightarrow 0, \quad s_{\alpha \beta} \rightarrow-1,
$$

the first doublet $\left(\Phi_{a}\right)$ becomes SM-like and $\widehat{H}$ is the light Higgs boson $H_{1}\left(\widehat{H} \equiv H_{1}\right)$ with SM-like couplings to fermions and gauge bosons. All the other new physical Higgs-boson fields $\left(H^{ \pm}, \widetilde{H} \equiv-H_{\mathrm{h}}, H_{\mathrm{a}}\right)$ are in the second doublet $\Phi_{b}$. As a result, there is no mixing between fields from one doublet with those of the other doublet in the alignment limit. Vice versa, if $s_{\alpha \beta} \rightarrow 0$ (and $c_{\alpha \beta} \rightarrow 1$ ) the heavy Higgs boson $H_{\mathrm{h}}$ has SM-like couplings.

In order to give masses to the fermions in the 2HDM, one has different options to construct Yukawa interactions between the fermionic and scalar fields. In particular, one distinguishes four different types of $2 \mathrm{HDM}$ Yukawa terms. In type I, only $\Phi_{2}$ couples to fermions. In type II, the down-type quarks as well as the leptons couple to $\Phi_{1}$, while the up-type quarks couple to $\Phi_{2}$. Then, in type Y (or type flipped), $\Phi_{2}$ couples to up-type quarks and charged leptons, while $\Phi_{1}$ couples to down-type quarks, and finally, we have type X (or type lepton specific), where $\Phi_{2}$ couples to quarks, while $\Phi_{1}$ couples to charged leptons. Within this work we focus on type II. This corresponds to the configuration that is realized in the Minimal Supersymmetric SM. As already mentioned in the beginning of this section, we impose a discrete $Z_{2}$ symmetry in order to avoid tree-level FCNC [34, 35]. For the type II $2 \mathrm{HDM}$, down-type quarks and charged leptons need to be odd under this $Z_{2}$ transformation, i.e. $\Phi_{1} \rightarrow-\Phi_{1}, d_{R} \rightarrow-d_{R}, \ell_{R} \rightarrow-\ell_{R}$, while all other fields remain unchanged. Here, the fields $d_{R}$ and $\ell_{R}$ are the right-handed, down-type quarks and the right-handed charged leptons. All fermions except for the top-quark are taken to be massless such that our results are valid for all types of 2HDM Yukawa terms. This is only justified for small values of $t_{\beta}$. Furthermore, we neglect flavour mixing in the following.

As new input parameters of the extended Higgs sector, we have the masses of the heavy, charged and pseudoscalar Higgs bosons

$$
M_{H_{\mathrm{h}}}, \quad M_{H_{\mathrm{c}}}, \quad M_{H_{\mathrm{a}}}
$$

as well as the soft-breaking scale

$$
M_{\mathrm{sb}}^{2}=\frac{m_{12}^{2}}{\cos \beta \sin \beta}
$$

and the mixing angles $\alpha$ and $\beta$, which we express through

$$
c_{\alpha \beta} \quad \text { and } \quad t_{\beta}=\tan \beta=\frac{v_{2}}{v_{1}} .
$$


Several limits of the new parameters can be defined. Beside the already mentioned alignment limit, the decoupling limit will be studied in this work. In this limit, not only $c_{\alpha \beta}$ is equal to zero, but in addition, all new mass scales of the $2 \mathrm{HDM}$ are much larger than the electroweak scale $[22,36]$.

\section{Renormalization of the mixing angles $\alpha$ and $\beta$}

In this section, we consider different renormalization schemes for the mixing angles $\alpha$ and $\beta$. We distinguish the $\overline{\mathrm{MS}}$ renormalization, which leads to scale-dependent amplitudes, and the on-shell, $p^{*}$ as well as two process-dependent schemes. The latter guarantee scaleindependent amplitudes. In the process considered in this work, the parameter $M_{\mathrm{sb}}$ does not enter at LO. Thus, it does not require renormalization unless the running is taken into account in the $\overline{\mathrm{MS}}$ renormalization scheme.

In section 5, we will show numerical results for these renormalization schemes. Having different renormalization schemes at hand can be valuable in order to estimate the residual uncertainty due to unknown higher order corrections.

$\overline{\text { MS }}$ renormalization scheme. An $\overline{\mathrm{MS}}$ renormalization of the mixing angles $\alpha$ and $\beta$ has been defined in ref. [26]. Here, the $\overline{\mathrm{MS}}$ counterterm $\delta \beta^{\overline{\mathrm{MS}}}$ is defined at the $\tau-H_{a}-\tau$ vertex, and the $\overline{\mathrm{MS}}$ counterterm $\delta \alpha^{\overline{\mathrm{MS}}}$ is defined at the $\tau-H_{l}-\tau$ vertex. The counterterms $\delta \alpha^{\overline{\mathrm{MS}}}$ and $\delta \beta^{\overline{\mathrm{MS}}}$ are required to render all amplitudes finite. The treatment of tadpoles requires special care when using an $\overline{\mathrm{MS}}$ renormalization of the mixing angles $\alpha$ and $\beta$ in order to guarantee gauge-independent amplitudes. The tadpoles have been treated in the FJ tadpole scheme, which has been introduced by Fleischer and Jegerlehner for the SM in ref. [37] and which was extended for the 2HDM, and also for a general Higgs sector, in ref. [38] and ref. [26]. In the SM, the FJ tadpole scheme corresponds to the $\beta_{t}$ scheme of ref. [39]. In this approach, the tadpole contributions are not absorbed into bare physical parameters, which intrinsically assures gauge-independent physical counterterms. A renormalization of the soft-breaking scale $M_{\mathrm{sb}}$ in the $\overline{\mathrm{MS}}$ scheme at the $H_{c}-H_{h}-H_{c}$ vertex was introduced in ref. [26]. For an $\overline{\mathrm{MS}}$ renormalization of $M_{\mathrm{sb}}$ see also ref. [40]. At next-to-leading order, the counterterms $\delta \alpha$ and $\delta \beta$ of the $2 \mathrm{HDM}$ can be obtained from the off-diagonal elements of the field-renormalization constants of the Higgs- and Goldstoneboson fields [41]. In particular, for a gauge-independent $\overline{\mathrm{MS}}$ renormalization, this relation reads

$$
\delta \alpha^{\overline{\mathrm{MS}}}=\frac{\delta Z_{H_{\mathrm{h}}}^{\overline{\mathrm{MS}} H_{1}}-\delta Z_{H_{1} H_{\mathrm{h}}}^{\overline{\mathrm{MS}}}}{4}, \quad \delta \beta^{\overline{\mathrm{MS}}}=\frac{\delta Z_{G_{0} H_{\mathrm{a}}}^{\overline{\mathrm{MS}}}-\delta Z_{H_{\mathrm{a}} G_{0}}^{\overline{\mathrm{MS}}}}{4},
$$

where the $Z$-factors are defined by

$$
\begin{aligned}
& \left(\begin{array}{c}
H_{\mathrm{h}, \mathrm{B}} \\
H_{\mathrm{l}, \mathrm{B}}
\end{array}\right)=\left(\begin{array}{cc}
1+\frac{1}{2} \delta Z_{H_{\mathrm{h}} H_{\mathrm{h}}} & \frac{1}{2} \delta Z_{H_{\mathrm{h}} H_{\mathrm{l}}} \\
\frac{1}{2} \delta Z_{H_{\mathrm{l}} H_{\mathrm{h}}} & 1+\frac{1}{2} \delta Z_{H_{\mathrm{l}} H_{\mathrm{l}}}
\end{array}\right)\left(\begin{array}{c}
H_{\mathrm{h}} \\
H_{\mathrm{l}}
\end{array}\right), \\
& \left(\begin{array}{c}
G_{0, \mathrm{~B}} \\
H_{\mathrm{a}, \mathrm{B}}
\end{array}\right)=\left(\begin{array}{cc}
1+\frac{1}{2} \delta Z_{G_{0} G_{0}} & \frac{1}{2} \delta Z_{G_{0} H_{\mathrm{a}}} \\
\frac{1}{2} \delta Z_{H_{\mathrm{a}} G_{0}} & 1+\frac{1}{2} \delta Z_{H_{\mathrm{a}} H_{\mathrm{a}}}
\end{array}\right)\left(\begin{array}{l}
G_{0} \\
H_{\mathrm{a}}
\end{array}\right),
\end{aligned}
$$


where the subscript $B$ denotes a bare field. For the renormalization conditions of the $Z$-factors we refer to ref. [26].

The choice of an $\overline{\mathrm{MS}}$ renormalization leads to scale-dependent renormalized amplitudes. In order to account for the different scales in the new Higgs sector, we use the average

$$
\mu_{0}=\left\{\begin{array}{lll}
\frac{M_{H_{1}}+M_{H_{\mathrm{h}}}+M_{H_{\mathrm{a}}}+2 M_{H_{\mathrm{c}}}+M_{\mathrm{sb}}}{6}, & \text { if } \quad M_{\mathrm{sb}} \neq 0 \\
\frac{M_{H_{1}}+M_{H_{\mathrm{h}}}+M_{H_{\mathrm{a}}}+2 M_{H_{\mathrm{c}}}}{5}, & \text { if } \quad M_{\mathrm{sb}}=0
\end{array}\right.
$$

as a central renormalization scale, see refs. $[28,29]$ for a similar scale choice.

In addition to the explicit scale dependence of the amplitude, the renormalization scale dependence, i.e. the running of the parameters, can be taken into account by solving a system of coupled differential equations:

$$
\begin{aligned}
& \frac{\partial \alpha}{\partial \ln \left(\mu^{2}\right)}=\mathrm{B}_{\alpha}\left(\alpha(\mu), \beta(\mu), M_{\mathrm{sb}}(\mu)\right), \\
& \frac{\partial \beta}{\partial \ln \left(\mu^{2}\right)}=\mathrm{B}_{\beta}\left(\alpha(\mu), \beta(\mu), M_{\mathrm{sb}}(\mu)\right), \\
& \frac{\partial M_{\mathrm{sb}}}{\partial \ln \left(\mu^{2}\right)}=\mathrm{B}_{M_{\mathrm{sb}}}\left(\alpha(\mu), \beta(\mu), M_{\mathrm{sb}}(\mu)\right) .
\end{aligned}
$$

The explicit expression for the functions $\mathrm{B}_{\alpha}, \mathrm{B}_{\beta}$ and $\mathrm{B}_{M_{\mathrm{sb}}}$ can directly be obtained from the pole part of the corresponding counterterms and are lengthy in the non-alignment limit.

In the $\overline{\mathrm{MS}}$ scheme the explicit values of the input parameter $c_{\alpha \beta}$ and $t_{\beta}$ need to be defined at a given scale $\mu$. We use two different choices of the scale at which we define these values: the averaged scale $\mu_{0}$ of eq. (3.4) as well as the vacuum expectation value $v$. For the latter choice we run the values from the scale $v$ to the scale $\mu_{0}$ with the help of the renormalization group equations (RGE) (3.5). We perform the scale variation $\mu_{0} / 2$ and $2 \mu_{0}$ and run the values of $c_{\alpha \beta}$ and $t_{\beta}$ to these corresponding scales. All results in the $\overline{\mathrm{MS}}$ scheme in section 5 are presented with the parameters defined at $\mu_{0}$. In appendix $\mathrm{C}$ we also compare the different $\overline{\mathrm{MS}}$ schemes for the $M^{*}$ benchmark scenarios, which will be introduced in section 5 .

On-shell renormalization scheme. We have also considered the on-shell (OS) scheme, where the renormalized mixing angles are defined such that they diagonalize the radiatively corrected Higgs mass matrices introduced in section 2. This connects the counterterms of the mixing angles $\alpha$ and $\beta$ to the off-diagonal terms of the on-shell two-point functions of the CP-even Higgs bosons and of the CP-odd Higgs boson and the neutral Goldstone boson, respectively. The mixing two-point functions are evaluated on-shell, which guarantees scale independence of the rotation matrices in eq. (2.4), see refs. [42, 43]. However, using finite parts of the mixing two-point functions to define the renormalization constants of $\alpha$ and $\beta$ leads to gauge-dependent renormalization constants in addition to the gauge-dependent tadpole terms discussed in ref. [26]. The problem has been overcome in ref. [38] using the pinch technique [42-55]. Recently, it has been treated in a more general framework in ref. [27] within the background field method (BFM), leading to the same result. In the 
pinch technique the two-point function $\widetilde{\Sigma}_{S S^{\prime}}\left(p^{2}\right)$ of two scalars $S$ and $S^{\prime}$ for the $\mathrm{R}_{\xi}$-gauge with $\xi=1$ is given by

$$
\widetilde{\Sigma}_{S S^{\prime}}\left(p^{2}\right)=\Sigma_{S S^{\prime}}^{1 \mathrm{PI}}\left(p^{2}\right)+\Sigma_{S S^{\prime}}^{\mathrm{add}}\left(p^{2}\right)
$$

where $\Sigma_{S S^{\prime}}^{1 \mathrm{PI}}\left(p^{2}\right)$ is the one-particle irreducible (1PI) mixing two-point function. In the $2 \mathrm{HDM}$ the additional contribution $\Sigma_{S S^{\prime}}^{\mathrm{add}}\left(p^{2}\right)$ reads [38]

$$
\begin{gathered}
\Sigma_{H_{\mathrm{h}} H_{1}}^{\mathrm{add}}\left(p^{2}\right)=\frac{g^{2} s_{\alpha \beta} c_{\alpha \beta}}{32 \pi^{2} c_{W}^{2}}\left(p^{2}-\frac{M_{H_{\mathrm{h}}}^{2}+M_{H_{1}}^{2}}{2}\right)\left\{B_{0}\left(p^{2} ; M_{Z}^{2}, M_{Z}^{2}\right)-B_{0}\left(p^{2} ; M_{Z}^{2}, M_{H_{\mathrm{a}}}^{2}\right)\right. \\
\left.\quad+2 c_{W}^{2}\left[B_{0}\left(p^{2} ; M_{W}^{2}, M_{W}^{2}\right)-B_{0}\left(p^{2} ; M_{W}^{2}, M_{H_{\mathrm{c}}}^{2}\right)\right]\right\} \\
\Sigma_{G_{0} H_{\mathrm{a}}}^{\mathrm{add}}\left(p^{2}\right)=\frac{g^{2} s_{\alpha \beta} c_{\alpha \beta}}{32 \pi^{2} c_{W}^{2}}\left(p^{2}-\frac{M_{H_{\mathrm{a}}}^{2}}{2}\right)\left\{B_{0}\left(p^{2} ; M_{Z}^{2}, M_{H_{1}}^{2}\right)-B_{0}\left(p^{2} ; M_{Z}^{2}, M_{H_{\mathrm{h}}}^{2}\right)\right\}
\end{gathered}
$$

with the cosine of the weak mixing angle $c_{W}$, the gauge coupling $g$ and the one-loop, scalar, two-point integral $B_{0}[56,57]$.

The counterterms to the mixing angles are then given by

$$
\begin{aligned}
\delta \alpha & =\frac{\operatorname{Re}\left[\widetilde{\Sigma}_{H_{\mathrm{h}} H_{1}}\left(M_{H_{\mathrm{h}}}^{2}\right)+\widetilde{\Sigma}_{H_{\mathrm{h}} H_{1}}\left(M_{H_{1}}^{2}\right)+2 t_{H_{\mathrm{h}} H_{1}}\right]}{2\left(M_{H_{\mathrm{h}}}^{2}-M_{H_{1}}^{2}\right)}, \\
\delta \beta & =-\frac{\operatorname{Re}\left[\widetilde{\Sigma}_{G_{0} H_{\mathrm{a}}}(0)+\widetilde{\Sigma}_{G_{0} H_{\mathrm{a}}}\left(M_{H_{\mathrm{a}}}^{2}\right)+2 t_{G_{0} H_{\mathrm{a}}}\right]}{2 M_{H_{\mathrm{a}}}^{2}},
\end{aligned}
$$

where the tadpole counterterms $t_{H_{\mathrm{h}} H_{1}}$ and $t_{G_{0} H_{\mathrm{a}}}$ are given in the appendix of ref. [26]. In an alternative on-shell scheme, the counterterm $\delta \beta$ can also be defined through the charged Higgs-boson-Goldstone-boson mixing two-point functions.

These schemes can be expected to produce small perturbative corrections to physical observables, because the finite counterterms of the mixing angles $\alpha$ and $\beta$ cancel large terms originating from the Z-factors of the neutral, scalar sector and in the pseudoscalar/charged sector as mentioned in ref. [27].

$\boldsymbol{p}^{*}$ renormalization scheme. The $p^{*}$ scheme is derived from the same mixing two-point functions of eq. (3.6), but computed at the external momentum

$$
\left(p^{*}\right)^{2}=\frac{M_{S}^{2}+M_{S^{\prime}}^{2}}{2}
$$

such that the rotation matrices in eq. (2.4) also remain scale-independent, see refs. [42, 43]. The additional terms in eqs. (3.7) vanish and the two-point functions in eq. (3.6) are equal to the 1PI two-point functions in the $\mathrm{R}_{\xi}$-gauge for $\xi=1$. This results in the counterterms 

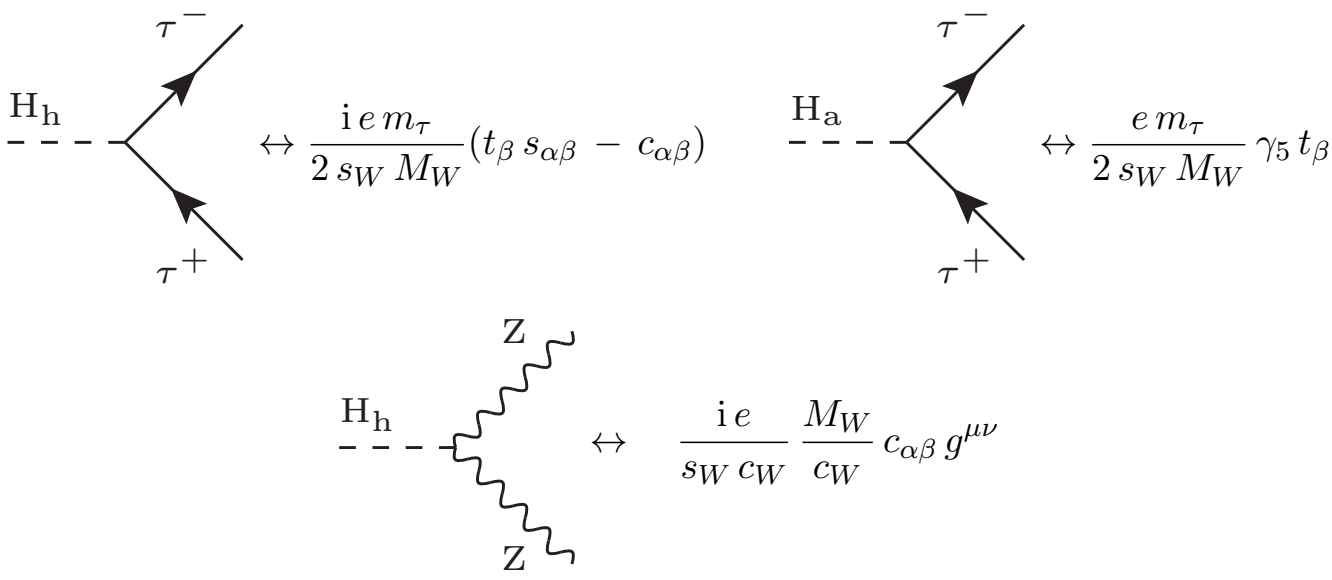

Figure 1. The vertices that are used for the determination of the counterterms $\delta \beta, \delta \alpha$ or $\delta \phi$ with $\phi=\alpha-\beta$ are shown with their corresponding Feynman rules. The symbol $e$ is the elementary electric charge, $M_{W}\left(m_{\tau}\right)$ is the mass of the $W$ boson $\left(\tau\right.$ lepton) and $s_{W}=\sqrt{1-c_{W}^{2}}$.

of the mixing angles

$$
\begin{gathered}
\delta \alpha=\frac{\operatorname{Re}\left[\widetilde{\Sigma}_{H_{\mathrm{h}} H_{1}}\left(\frac{M_{H_{\mathrm{h}}}^{2}+M_{H_{1}}^{2}}{2}\right)+t_{H_{\mathrm{h}} H_{1}}\right]}{M_{H_{\mathrm{h}}}^{2}-M_{H_{1}}^{2}}=\frac{\operatorname{Re}\left[\Sigma_{H_{\mathrm{h}} H_{1}}\left(\frac{M_{H_{\mathrm{h}}}^{2}+M_{H_{1}}^{2}}{2}\right)+t_{H_{\mathrm{h}} H_{1}}\right]_{\xi=1}}{M_{H_{\mathrm{h}}}^{2}-M_{H_{1}}^{2}}, \\
\delta \beta=-\frac{\operatorname{Re}\left[\widetilde{\Sigma}_{G_{0} H_{\mathrm{a}}}\left(\frac{M_{H_{\mathrm{a}}}^{2}}{2}\right)+t_{G_{0} H_{\mathrm{a}}}\right]}{M_{H_{\mathrm{a}}}^{2}}=-\frac{\operatorname{Re}\left[\Sigma_{G_{0} H_{\mathrm{a}}}\left(\frac{M_{H_{\mathrm{a}}}^{2}}{2}\right)+t_{G_{0} H_{\mathrm{a}}}\right]_{\xi=1}}{M_{H_{\mathrm{a}}}^{2}} .
\end{gathered}
$$

Process-dependent renormalization scheme 1. A process-dependent renormalization of the parameters $\alpha$ and $\beta$ can be obtained by imposing renormalization conditions on the physical decay processes $H_{h} \rightarrow \tau^{+} \tau^{-}$and $H_{a} \rightarrow \tau^{+} \tau^{-}$[38]. The two counterterms $\delta \alpha$

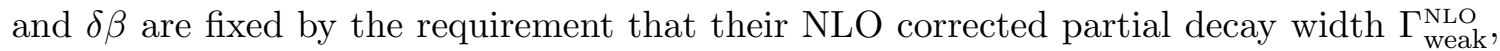
which contains weak corrections only, is equal to the LO partial decay width $\Gamma^{\mathrm{LO}}$, i.e.

$$
\Gamma_{\text {weak }}^{\mathrm{NLO}}\left(H_{h} \rightarrow \tau^{+} \tau^{-}\right)=\Gamma^{\mathrm{LO}}\left(H_{h} \rightarrow \tau^{+} \tau^{-}\right) \quad \text { and } \quad \Gamma_{\text {weak }}^{\mathrm{NLO}}\left(H_{a} \rightarrow \tau^{+} \tau^{-}\right)=\Gamma^{\mathrm{LO}}\left(H_{a} \rightarrow \tau^{+} \tau^{-}\right) .
$$

Since both vertices $\left(\tau-H_{h}-\tau\right.$ and $\left.\tau-H_{a}-\tau\right)$ depend on $t_{\beta}$, see figure 1 for illustration, the determination of both counterterms $\delta \alpha$ and $\delta \beta$ requires the solution of a linear system of equations. First, $\delta \beta$ can be determined from the $\tau-H_{a}-\tau$ vertex, which depends only on $t_{\beta}$. Then, the result needs to be inserted into the $\tau-H_{h}-\tau$ vertex for the determination of $\delta \alpha$. We refer to this scheme as proc1 in the following.

If the additional 2HDM particles are discovered, one can expect that these two processes can be measured precisely due to the leptonic final states. Therefore, they are in principle well suited for the determination of the parameters $\alpha$ and $\beta$. Due to the choice of the renormalization condition, no higher order electroweak corrections are present in the 
determination of $\alpha$ and $\beta$. Process-dependent renormalization may lead to large perturbative corrections, since the higher order corrections to the processes used for renormalization will appear in all other processes. Such a behaviour was, for example, observed for the decay process $H^{ \pm} \rightarrow W^{ \pm} H_{1}$ in ref. [38]. For light and heavy Higgs-boson production through gluon fusion, however, process-dependent renormalization leads to results similar to those obtained in the OS or $p^{*}$ scheme, as we will see in section 5 .

Process-dependent renormalization scheme 2. Unlike the previously discussed physical renormalization conditions, which rely on the partial decay widths of two leptonic Higgs-boson decays, we consider a process dependent renormalization, which is based on the two vertices $\tau-H_{a}-\tau$ and $Z-H_{h}-Z$ in the following. Studying a potential decay of a heavy Higgs boson into two $Z$-bosons is experimentally less clean, since the unstable $Z$-bosons can further decay into a variety of other particles. In addition, its LO contribution is very small in physically relevant scenarios close to the alignment limit. Nevertheless, it turns out, as we will see in section 5 , that in the calculation of the processes $g+g \rightarrow H_{1}$ and $g+g \rightarrow H_{\mathrm{h}}$, this scheme leads to higher order corrections of moderate size.

As renormalization condition, we require that the purely weak corrected $\tau-H_{a}-\tau$ vertex is equal to its leading order value in order to fix the counterterm $\delta \beta$. We impose a similar condition on the $Z-H_{h}-Z$ vertex in order to fix the counterterm $\delta \phi$. Note that due to our choice of parameters, $t_{\beta}$ and $c_{\alpha \beta}$, it is more convenient to renormalize $\phi=\alpha-\beta$ rather than $\alpha$. The $Z-H_{h}-Z$ vertex is proportional to $g^{\mu \nu}$ at LO, see figure 1 . Computing higher order corrections to this vertex leads to a richer tensor structure, which also contains combinations of the four-momenta of the external $Z$-bosons. As renormalization condition for the counterterm $\delta \phi$, we require that the coefficient in front of $g^{\mu \nu}$ of the $Z-H_{h}-Z$ amplitude is equal to its leading order value similar to the renormalization of the electric charge in QED. The coefficient in front of $g^{\mu \nu}$ can be extracted from the amplitude by using a suitable projector. This scheme will be called proc2 in the following. The use of the vertices $\tau-H_{a}-\tau$ and $Z-H_{h}-Z$ has the advantage that one has a separate condition for each of the two counterterms $\delta \beta$ and $\delta \phi$, i.e. the value of $\delta \phi$ is not influenced by the renormalization condition for $\delta \beta$, contrary to the previously discussed proc1 scheme.

\section{Calculation}

The partonic, leading order cross section for the Higgs-boson production process through gluon fusion reads

$$
\begin{aligned}
\sigma_{2 \mathrm{HDM}}^{\mathrm{LO}}(g+g \rightarrow H) & =\frac{G_{F} M_{H}^{2} \alpha_{s}^{2}}{128 \sqrt{2} \pi}\left|A_{H}^{\mathrm{LO}}\right|^{2} \delta\left(s-M_{H}^{2}\right) \\
& \equiv \hat{\sigma}^{\mathrm{LO}} M_{H}^{2} \delta\left(s-M_{H}^{2}\right),
\end{aligned}
$$

where $s$ is the square of the sum of the external gluon $(g)$ momenta, $\alpha_{s}$ is the strong coupling constant and $G_{F}$ is the Fermi coupling constant. The leading order amplitude $A^{\mathrm{LO}}$ reads

$$
A_{H}^{\mathrm{LO}}=\sum_{q} c_{H, q}^{2 \mathrm{HDM}} \frac{1}{\tau_{q}}\left[1+\left(1-\frac{1}{\tau_{q}}\right) f\left(\tau_{q}\right)\right] \quad \text { with } \quad \tau_{q}=\frac{M_{H}^{2}}{4 M_{q}^{2}},
$$


where the index $q$ runs over all quark flavours and

$$
f\left(\tau_{q}\right)= \begin{cases}\arcsin ^{2} \sqrt{\tau_{q}}, & \text { if } \quad \tau_{q} \leq 1, \text { i.e. } q=t \\ -\frac{1}{4}\left[\ln \left(\frac{1+\sqrt{1-1 / \tau_{q}}}{1-\sqrt{1-1 / \tau_{q}}}\right)-\mathrm{i} \pi\right]^{2}, & \text { if } \quad \tau_{q}>1, \text { i.e. } q=u, d, s, c, b .\end{cases}
$$

The symbol $H$ in eq. (4.1) stands for the external Higgs boson, which can be either the light $\left(H=H_{\mathrm{l}}\right)$ or the heavy $\left(H=H_{\mathrm{h}}\right)$ neutral Higgs boson of the $2 \mathrm{HDM}$; equally, $M_{H}$ is the Higgs-boson mass, which can be either $M_{H}=M_{H_{1}}$ or $M_{H}=M_{H_{\mathrm{h}}}$. The symbol $M_{q}$ is the mass of the internal quark, which in general can be either the up $(u)$, down $(d)$, strange $(s)$, charm $(c)$, bottom $(b)$ or top $(t)$ quark. The coefficient $c_{H, q}^{2 H D M}$ in eq. (4.2) originates from the Higgs-boson-quark coupling and is in the 2HDM, type II different for up- and down-type quarks and also different for the production of a light or a heavy, neutral Higgs boson:

$$
\begin{array}{lll}
c_{H_{1}, q}^{2 \mathrm{HDM}}=c_{\alpha \beta} / t_{\beta}-s_{\alpha \beta} & \text { and } \quad & c_{H_{\mathrm{h}}, q}^{2 \mathrm{HDM}}=c_{\alpha \beta}+s_{\alpha \beta} / t_{\beta}
\end{array} \quad \text { for } \quad q \in\{u, c, t\},
$$

The production of the SM Higgs boson corresponds to the case where $c_{H, q}^{2 \mathrm{HDM}}$ is equal to one. We consider only the top quark as massive and all other fermions as massless, i.e. only the case $q=t$ contributes to eq. (4.1). In this case only $c_{H_{1}, t}^{2 \mathrm{HDM}}$ or $c_{H_{\mathrm{h}}, t}^{2 \mathrm{HDM}}$ of eq. (4.4) contributes to eq. (4.2). For simplicity we will drop the label $t$ in the following, i.e. $c_{H_{1}}^{2 \mathrm{HDM}} \equiv c_{H_{1}, t}^{2 \mathrm{HDM}}$ and $c_{H_{\mathrm{h}}}^{2 \mathrm{HDM}} \equiv c_{H_{\mathrm{h}}, t}^{2 \mathrm{HDM}}$. In particular, $c_{H_{1}}^{2 \mathrm{HDM}}$ of eq. (4.4) becomes one, i.e. SM-like, in the alignment limit given in eq. (2.8). One can also find a combination of $c_{\alpha \beta}$ and $t_{\beta}$ such that $c_{H}^{2 H D M}$ becomes arbitrary small or even zero. In this case, the two-loop, electroweak corrected process becomes the true leading order process.

There are no real corrections which have to be determined when computing the NLO electroweak corrections for this process. Consequently, one can straightforwardly apply the results for the electroweak percentage correction, which has been obtained for the cross section of the production process $g+g \rightarrow H$ in eq. (4.1), to the partial decay width of the process $H \rightarrow g+g$. The partial decay width and the production cross section are related by

$$
\Gamma_{\mathrm{LO}}(H \rightarrow g+g)=\frac{8 M_{H}^{3}}{\pi^{2}} \hat{\sigma}_{\mathrm{LO}}(g+g \rightarrow H) .
$$

The Feynman rules for the 2HDM of type II, which has been defined in section 2, have been produced in an automated way with the help of FeynRules [58]. The one- and two-loop diagrams have been calculated with QGS, which is an extension of GraphShot [59]. It generates the Feynman diagrams with QGRAF [60] and performs algebraic manipulations of the amplitudes and loop integrals with the help of Form [61, 62]. The program QGS performs the standard Dirac algebra operations and projects the expressions onto form factors. In addition, it removes reducible scalar products and uses integration-by-parts identities in order to simplify tadpole contributions. Finally, it reduces the number of loop integrals by means of symmetrization. Further details regarding these techniques can be found in ref. [31]. After these manipulations, one remains with a two-loop amplitude that 

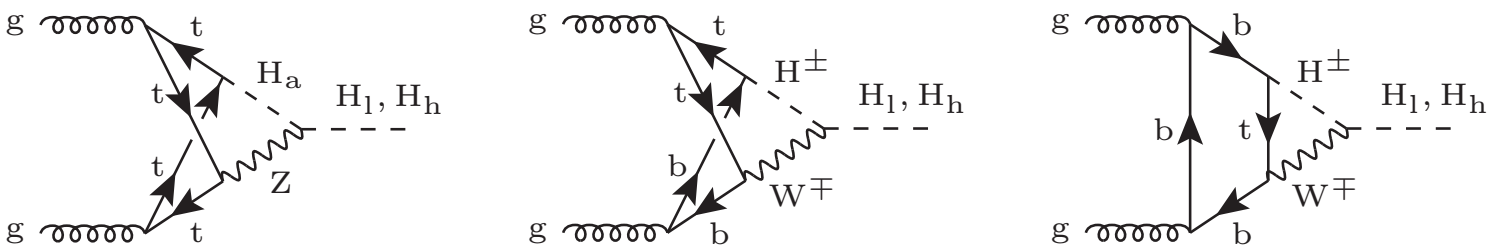

Figure 2. Sample diagrams that do not appear in light Higgs-boson production in the alignment limit are shown. In the second and third diagram, the bottom quarks are considered to be massless. For light Higgs-boson production the contribution to the amplitude originating from these three diagrams is proportional to $\cos (\alpha-\beta)$. This is the reason why they vanish in the alignment limit.

requires numerical evaluation, which is done with a Fortran code. In particular, the twoloop integrals are evaluated in Feynman-parametric space with the help of a Fortran library. For the numerical integral evaluation of the two-loop massive diagrams, the library uses the methods of refs. [63, 64] for self-energies and of refs. [31, 65-67] for vertex functions.

Compared to the production of a light, neutral, SM-like Higgs boson $H_{1}$ in gluon fusion in the alignment limit, which has been addressed already briefly in ref. [26], the non-alignment limit case and the production of a heavy, neutral Higgs boson $H_{\mathrm{h}}$ is computationally more involved. While the calculation of the integrals for the production of the SM-like Higgs boson $H_{1}$ in the alignment limit can be completely traced back to the calculation of the pure SM case, which was discussed in refs. [30, 31], new integral structures appear in the general case. They are generated by new diagrams, a sample of which is shown in figure 2. The first diagram is non-planar and leads to a new rank 2 integral. In the SM and in the alignment limit, this diagram only exists with two Z-bosons. The same type of diagram exists for the W-bosons and the charged Higgs bosons (second diagram of figure 2). Since different types of fermions appear in this diagram, it leads to rank 3 integrals, and hence, the calculation becomes even more involved. In the SM, it was possible to cancel the rank 3 integrals since there were fewer different bosonic masses in the denominators. A new planar diagram, which appears in the general case of the 2HDM, is depicted at the rightmost side of figure 2. After performing the obvious reduction as explained in ref. [31], some diagrams lead to collinear divergent structures; the new ones, not present in the alignment limit, are shown in figure 3. In particular, the second non-planar diagram of figure 2 leads to the more complicated collinear structure depicted in the second diagram of figure 3. More details regarding the cancellation of these new collinear singularities are given in section 4.1 .

In section 5, we provide numerical results for the NLO, electroweak corrections to the production processes $g+g \rightarrow H_{\mathrm{l}}$ and $g+g \rightarrow H_{\mathrm{h}}$ for various 2HDM scenarios. We define the NLO corrections in terms of the K-factor

$$
\begin{aligned}
\left|A_{H}^{\mathrm{NLO}, \mathrm{EW}}\right|^{2} & =\left|A_{H}^{(1)}+A_{H}^{(2)}\right|^{2} \\
& =\left|A_{H}^{(1)}\right|^{2}+2 \operatorname{Re}\left(A_{H}^{(1)} A_{H}^{(2) *}\right)+\mathcal{O}\left(\left(G_{F} M_{W}^{2}\right)^{2}\right) \equiv\left|A_{H}^{(1)}\right|^{2} K_{\mathrm{EW}}^{\mathrm{NLO}},
\end{aligned}
$$

using the one-loop $A_{H}^{(1)}$ and two-loop $A_{H}^{(2)}$ contributions to the amplitude with the normalization defined in eq. (4.1). 

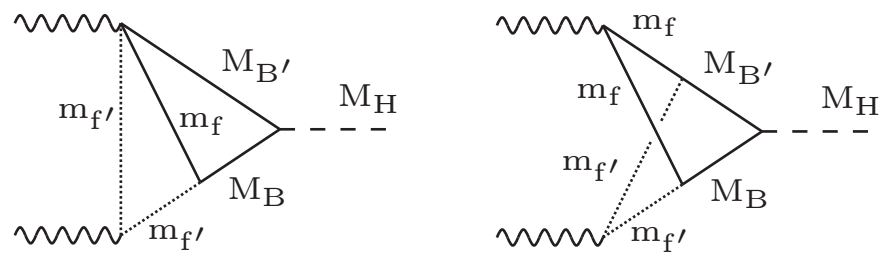

Figure 3. Collinear structures that do not appear in the alignment limit are shown. Massless particles are marked as wavy or dotted lines, while solid lines denote massive particles.

If the coefficient $c_{H_{1}}^{2 \mathrm{HDM}}$ or $c_{H_{\mathrm{h}}}^{2 \mathrm{HDM}}$ of eq. (4.4) becomes very small, the one-loop amplitude $A_{H}^{(1)}$ is very small too, and the two-loop amplitude $A_{H}^{(2)}$ becomes the true leading order result. As discussed in the context of Higgs-boson production and decay with a fourth fermion generation [25], neglecting the term $\left|A_{H}^{(2)}\right|^{2}$ is no longer justified in this case, and we define a new K-factor

$$
\left|A_{H}^{\mathrm{NLO}, \mathrm{EW}}\right|^{2}=\left|A_{H}^{(1)}+A_{H}^{(2)}\right|^{2}=\left|A_{H}^{(1)}\right|^{2}+2 \operatorname{Re}\left(A_{H}^{(1)} A_{H}^{(2) *}\right)+\left|A_{H}^{(2)}\right|^{2} \equiv\left|A_{H}^{(1)}\right|^{2} \bar{K}_{\mathrm{EW}}^{\mathrm{NLO}} .
$$

Whenever $\bar{K}_{\mathrm{EW}}^{\mathrm{NLO}}$ differs significantly from $K_{\mathrm{EW}}^{\mathrm{NLO}}, \bar{K}_{\mathrm{EW}}^{\mathrm{NLO}}$ will be used in section 5 .

\subsection{Analytical and numerical tests}

We have performed several analytical as well as numerical tests to validate the new components of QGS, which are the 2HDM Feynman rules, automatic generation of Feynman diagrams and the appearances of new rank 2 and 3 integrals.

The ultraviolet (UV) structure of the new integrals as well as the consistency of the 2HDM Feynman rules has been tested by extracting all UV poles in dimensional regularization, as explained in ref. [31], and verifying their cancellation analytically.

Furthermore, the cancellation of collinear singularities can also be used to validate the implementation of the Feynman rules and the calculation of the new integral structures. Collinear singularities arise in some diagrams, if the external gluons couple to light fermions, but they have to cancel when summing up the contributions from all diagrams. These singularities have been regularized by fictitious small fermion masses and become manifest in terms of linear and quadratic logarithms. The two-loop electroweak amplitude is subdivided into the contribution that comes from the first and second generation of fermions and the contribution that originates from the third generation. The cancellation of the collinear divergent logarithms, which arise from the first and second generation of fermions, has been verified analytically. For the third generation of fermions, two new collinear structures, originating from the second and third diagram in figure 2, are presented in figure 3. Their analytic cancellation requires new integration-by-parts identities and a more complicated partial-fraction decomposition due to new denominators. All quadratically collinear divergent logarithms can then be cancelled analytically. The linearly collinear divergent logarithms have been treated in a numerical approach. Here, the logarithm is extracted analytically, but its coefficient is evaluated numerically. We have verified that the sum of all these coefficients cancels numerically. 


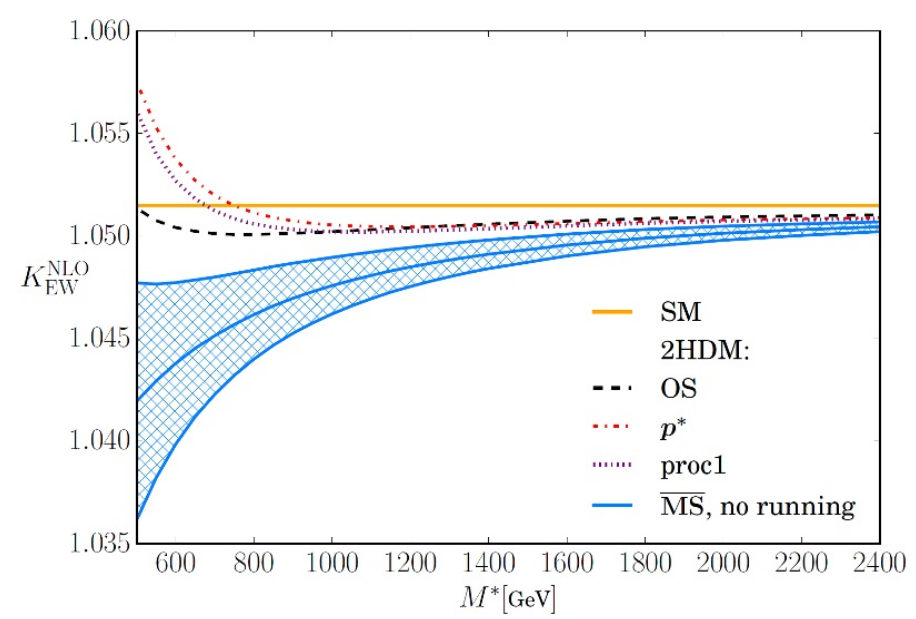

Figure 4. Decoupling scenario for the process $g+g \rightarrow H_{l}$ as defined in eqs. (4.8).

Another way to verify our implementation of the $2 \mathrm{HDM}$ is to test the behaviour of the NLO electroweak corrections in different limits. In the decoupling limit introduced in the end of section 2, all new Higgs-boson masses are much larger than the electroweak scale, such that the new particles decouple from the SM. Therefore, the NLO corrections in the 2HDM should approach those of the SM. Figure 4 shows a decoupling scenario for $g+g \rightarrow H_{1}$. The $2 \mathrm{HDM}$ parameters have been chosen as

$$
M_{H_{\mathrm{h}}}=M_{H_{\mathrm{a}}}=M_{H_{\mathrm{c}}}=M_{\mathrm{sb}}=M^{*}, \quad c_{\alpha \beta}=0, \quad t_{\beta}=2,
$$

where all masses are set equal to the scale of new physics $M^{*}$, because perturbativity requires the mass splitting to be smaller than $v^{2} / M^{*}$ [36]. At $M^{*}=2400 \mathrm{GeV}$, the electroweak corrections in the $2 \mathrm{HDM}$ have almost approached those of the SM. In addition, we observe that the scale dependence of the $\overline{\mathrm{MS}}$-renormalized corrections shrinks, cf. appendix A for the analytic formula. The proc2 renormalization has not been taken into account: since the LO of its defining process $H_{\mathrm{h}} \rightarrow Z Z$ vanishes in the alignment limit, it cannot be expected to show proper decoupling behaviour.

Furthermore, several one-loop processes and all finite NLO counterterms have been cross-checked against the implementation in RECOLA2 [27] to verify the implementation of the Feynman rules and the generation of one-loop diagrams.

\section{Results and discussion}

In this section, we present the numerical results of phenomenologically interesting scenarios for light and heavy Higgs-boson production in gluon fusion for the CP-conserving 2HDM. First, we consider the benchmark points (BP) in tables 1 and 2 in different renormalization schemes. The BPs a-1 and b-1 are taken from ref. [68] and they correspond to best-fit constraints on the triple Higgs couplings from the Higgs signal strengths. All other BPs are from the LHC Higgs cross section working group report [22] and are sample points of phenomenologically interesting allowed scenarios compatible with the 2HDM type II. 


\begin{tabular}{|c|c|c|c|c|c|c|c|c|}
\hline $\mathrm{BP}$ & $\frac{M_{H_{\mathrm{h}}}}{\mathrm{GeV}}$ & $\frac{M_{H_{\mathrm{a}}}}{\mathrm{GeV}}$ & $\frac{M_{H_{\mathrm{c}}}}{\mathrm{GeV}}$ & $\frac{m_{12}}{\mathrm{GeV}}$ & $t_{\beta}$ & $\frac{M_{\mathrm{sb}}}{\mathrm{GeV}}$ & $\frac{\left|\lambda_{i}^{\max }\right|}{4 \pi}$ & $\left|c_{H_{\mathrm{h}}}^{2 \mathrm{HDM}}\right|^{2}$ \\
\hline $2_{1 A}$ & 200.0 & 500.0 & 200.0 & 135.0 & 1.5 & 198.7 & 0.28 & 0.44 \\
\hline $2_{1 B}$ & 200.0 & 500.0 & 500.0 & 135.0 & 1.5 & 198.7 & 0.57 & 0.44 \\
\hline $2_{1 C}$ & 400.0 & 225.0 & 225.0 & 0.0 & 1.5 & 0.0 & 0.49 & 0.44 \\
\hline $2_{1 D}$ & 400.0 & 100.0 & 400.0 & 0.0 & 1.5 & 0.0 & 0.49 & 0.44 \\
\hline $3_{A 1}$ & 180.0 & 420.0 & 420.0 & 70.7 & 3.0 & 129.1 & 0.42 & 0.11 \\
\hline
\end{tabular}

Table 1. $2 \mathrm{HDM}$ benchmark points (BP) in the alignment limit, i.e. $c_{\alpha \beta}=0$, taken from ref. [22]. In the alignment limit, $\left|c_{H_{1}}^{2 \mathrm{HDM}}\right|^{2}$ is always 1.

\begin{tabular}{|c|c|c|c|c|c|c|c|c|c|c|}
\hline BP & $\frac{M_{H_{\mathrm{h}}}}{\mathrm{GeV}}$ & $\frac{M_{H_{\mathrm{a}}}}{\mathrm{GeV}}$ & $\frac{M_{H_{\mathrm{c}}}}{\mathrm{GeV}}$ & $\frac{m_{12}}{\mathrm{GeV}}$ & $t_{\beta}$ & $c_{\alpha \beta}$ & $\frac{M_{\mathrm{sb}}}{\mathrm{GeV}}$ & $\frac{\left|\lambda_{i}^{\mathrm{max}}\right|}{4 \pi}$ & $\left|c_{H_{\mathrm{l}}}^{2 \mathrm{HDM}}\right|^{2}$ & $\left|c_{H_{\mathrm{h}}}^{2 \mathrm{HDM}}\right|^{2}$ \\
\hline $\mathrm{a}-1$ & 700.0 & 700.0 & 670.0 & 424.3 & 1.5 & -0.091 & 624.5 & 0.16 & 0.87 & 0.57 \\
\hline $\mathrm{b}-1$ & 200.0 & 383.0 & 383.0 & 119.6 & 2.52 & -0.0346 & 204.2 & 0.30 & 0.97 & 0.19 \\
\hline $2_{2 A}$ & 500.0 & 500.0 & 500.0 & 187.1 & 7.0 & 0.28 & 500.0 & 0.64 & 1.00 & 0.02 \\
\hline $3_{B 1}$ & 200.0 & 420.0 & 420.0 & 77.8 & 3.0 & 0.3 & 142.0 & 0.44 & 1.11 & 0.0003 \\
\hline $3_{B 2}$ & 200.0 & 420.0 & 420.0 & 77.8 & 3.0 & 0.5 & 142.0 & 0.46 & 1.07 & 0.04 \\
\hline $4_{3}$ & 263.7 & 6.3 & 308.3 & 52.3 & 1.9 & 0.14107 & 81.5 & 0.35 & 1.13 & 0.14 \\
\hline $4_{4}$ & 227.1 & 24.7 & 226.8 & 58.4 & 1.8 & 0.14107 & 89.6 & 0.23 & 1.14 & 0.17 \\
\hline $4_{5}$ & 210.2 & 63.06 & 333.5 & 69.2 & 2.4 & 0.71414 & 116.2 & 0.31 & 1.00 & 0.18 \\
\hline
\end{tabular}

Table 2. 2HDM benchmark points (BP) outside the alignment limit taken from ref. [68] (a-1, b-1) and ref. [22].

Then, in order to analyze the influence of the mass splitting on the perturbative behaviour of the Higgs boson production processes, we move to a benchmark scenario where we keep all new Higgs boson masses fixed at $700 \mathrm{GeV}$ except for the heavy Higgs boson mass which is varied between $600 \mathrm{GeV}$ and $800 \mathrm{GeV}$. This scenario agrees with current experimental and theoretical exclusion limits [7, 69, 70].

For the numerical evaluation we use the following set of SM input parameters [3]:

$$
\begin{aligned}
G_{F} & =1.1663787 \cdot 10^{-5} \mathrm{GeV}^{-2}, \quad M_{W}=80.385 \mathrm{GeV}, \quad \Gamma_{W}=2.085 \mathrm{GeV}, \\
M_{Z} & =91.1876 \mathrm{GeV}, \quad \Gamma_{Z}=2.4952 \mathrm{GeV}, \quad M_{t}=173.1 \mathrm{GeV}, \quad \Gamma_{t}=1.41 \mathrm{GeV}, \\
M_{h} & \equiv M_{H_{1}}=125.09 \mathrm{GeV} .
\end{aligned}
$$

For the renormalization of the $W$ - and $Z$-boson masses we use the complex mass scheme [71-73]. In contrast to ref. [26], where the top-quark mass was renormalized onshell, here we also use the complex mass scheme [71-73] for the top-quark mass.

Benchmark points. According to the analysis of the LHC Higgs cross section working group [22], the BPs in tables 1 and 2 fulfill constraints like perturbativity and vacuum stability. In tables 1 and 2 we also provide the maximum of the couplings $\left|\lambda_{i}^{\max }\right| /(4 \pi)$ of the Higgs potential of eq. (2.2). Compared to the SM value $\lambda^{\mathrm{SM}} /(4 \pi)=0.02$, all $\left|\lambda_{i}^{\max }\right| /(4 \pi)$ values are large. This can potentially lead to large NLO corrections. 


\begin{tabular}{|c|c|c|c|c|c|c|}
\hline $\mathrm{BP}$ & $\frac{\hat{\sigma}_{\mathrm{LO}}^{\mathrm{LO}}}{\mathrm{fb}}$ & $K_{\mathrm{EW}}^{\mathrm{OS}}$ & $K_{\mathrm{EW}}^{p^{*}}$ & $K_{\mathrm{EW}}^{\mathrm{proc1}}$ & $K_{\mathrm{EW}}^{\mathrm{proc2}}$ & $K_{\mathrm{EW}}^{\overline{\mathrm{MS}}}$ \\
\hline $2_{1 A}$ & 51.856 & 1.053 & 1.063 & 1.101 & 1.037 & $0.994_{+0.342}^{-0.030}$ \\
\hline $2_{1 B}$ & 51.856 & 1.038 & 1.048 & 1.044 & 1.022 & $0.930_{+2.389}^{+0.066}$ \\
\hline $2_{1 C}$ & 51.856 & 1.043 & 1.044 & 1.099 & 1.055 & $1.126_{-0.007}^{-0.001}$ \\
\hline $2_{1 D}$ & 51.856 & 1.029 & 1.035 & 1.042 & 1.029 & $1.145_{-0.015}^{-0.012}$ \\
\hline $3_{A 1}$ & 51.856 & 1.041 & 1.040 & 1.045 & 1.041 & $1.118_{-0.010}^{-0.042}$ \\
\hline
\end{tabular}

Table 3. The NLO electroweak K-factors for the process $g+g \rightarrow H_{1}$ for the $2 \mathrm{HDM}$ benchmark points (BP) in the alignment limit are shown. They are defined in table 1 . The renormalization scale has been set to $\mu_{0}$ as defined in eq. (3.4) for the $\overline{\mathrm{MS}}$ scheme. The lower (upper) value of the $\overline{\mathrm{MS}}$ result corresponds to the change when taking $\mu_{0} / 2\left(2 \mu_{0}\right)$ as renormalization scale.

Table 3 contains the NLO electroweak K-factors for light Higgs-boson production in gluon fusion in different renormalization schemes in the alignment limit. For this process, we provide only the factor $K_{\mathrm{EW}}^{\mathrm{NLO}}$, because the difference between $K_{\mathrm{EW}}^{\mathrm{NLO}}$ and $\bar{K}_{\mathrm{EW}}^{\mathrm{NLO}}$ is tiny - at the per mille level. The OS, $p^{*}$ and the two process-dependent renormalization schemes produce similar results and mostly, the K-factors are comparable in size with the $\mathrm{SM}$ value $K_{\mathrm{EW}}^{\mathrm{SM}}=1.051$. For the $\overline{\mathrm{MS}}$-renormalized values, we have chosen $\mu_{0}$ according to eq. (3.4), different from the choice of the renormalization scale in ref. [26]. The values for $c_{\alpha \beta}$ and $t_{\beta}$ of the benchmark points in tables 1 and 2 are defined at the scale $\mu_{0}$. The uncertainty is obtained as described in section 3 by taking one half and twice the central value $\mu_{0}$ and running the parameters $c_{\alpha \beta}$ and $t_{\beta}$ to the corresponding scales. The results in the $\overline{\mathrm{MS}}$ scheme are different from the other results and the benchmark points $2_{1 A}$ and $2_{1 B}$ show a large scale dependence as already analyzed in ref. [26], where no running of the parameters was considered yet. The running results in an enhanced scale dependence for these two benchmark points.

For the remaining benchmark points with $c_{\alpha \beta} \neq 0$, the K-factors of the electroweak corrections to the process $g+g \rightarrow H_{1}$ are shown likewise in table 4. Again, the K-factors of the OS, $p^{*}$ and the two process-dependent renormalization schemes are close to the SM, while the $\overline{\mathrm{MS}}$ scheme leads to different results. Nevertheless, the electroweak corrections are always moderate in size, i.e. they are mostly below $5 \%$ compared to the LO production cross section.

We now turn to heavy, neutral Higgs-boson production $g+g \rightarrow H_{\mathrm{h}}$. There are two aspects that we have to consider when looking at this process. On the one hand, the LO production cross sections in tables 5 and 6 depend on the heavy Higgs-boson mass as well as on the coefficient $\left|c_{H_{\mathrm{h}}}^{2 \mathrm{HDM}}\right|^{2}$. On the other hand, the coefficients $\left|c_{H_{\mathrm{h}}}^{2 \mathrm{HDM}}\right|^{2}$ can be close to zero. This is different from the light Higgs-boson production case, where for all BPs the same light Higgs-boson mass enters, and where the coefficients $\left|c_{H_{1}}^{2 \mathrm{HDM}}\right|^{2}$, as given in table 2 , are always close to 1 or even equal to 1 , as it is the case in the alignment limit. For these reasons, the LO cross sections of the heavy Higgs-boson production can be very 


\begin{tabular}{|c|c|c|c|c|c|c|}
\hline $\mathrm{BP}$ & $\frac{\hat{\sigma}^{\mathrm{LO}}}{\mathrm{fb}}$ & $K_{\mathrm{EW}}^{\mathrm{OS}}$ & $K_{\mathrm{EW}}^{p^{*}}$ & $K_{\mathrm{EW}}^{\mathrm{proc1}}$ & $K_{\mathrm{EW}}^{\mathrm{proc} 2}$ & $K_{\mathrm{EW}}^{\overline{\mathrm{MS}}}$ \\
\hline $\mathrm{a}-1$ & 45.352 & 1.043 & 1.047 & 1.048 & 1.057 & $0.962_{+2.634}^{+0.042}$ \\
\hline $\mathrm{b}-1$ & 50.381 & 1.048 & 1.045 & 1.054 & 1.040 & $0.995_{+0.175}^{+0.002}$ \\
\hline $2_{2 A}$ & 51.856 & 1.017 & 1.018 & 1.015 & 1.017 & $1.006_{-}^{-0.871}$ \\
\hline $3_{B 1}$ & 57.601 & 1.039 & 1.038 & 1.039 & 1.032 & $1.072_{+0.079}^{-0.152}$ \\
\hline $3_{B 2}$ & 55.302 & 1.037 & 1.036 & 1.035 & 1.034 & $0.917_{+0.120}^{-0.054}$ \\
\hline $4_{3}$ & 58.733 & 1.042 & 1.043 & 1.038 & 1.036 & $1.126_{+0.030}^{-0.022}$ \\
\hline $4_{4}$ & 59.189 & 1.043 & 1.044 & 1.038 & 1.034 & $1.103_{+0.002}^{+0.002}$ \\
\hline $4_{5}$ & 51.603 & 1.037 & 1.036 & 1.026 & 1.070 & $1.045_{-0.075}^{+0.111}$ \\
\hline
\end{tabular}

Table 4. The NLO electroweak K-factors for the process $g+g \rightarrow H_{1}$ for the $2 \mathrm{HDM}$ benchmark points (BP) that are not in the alignment limit are presented. They are defined in table 2 . The renormalization scale has been set to $\mu_{0}$ as defined in eq. (3.4) for the $\overline{\mathrm{MS}}$ scheme. The lower (upper) value of the $\overline{\mathrm{MS}}$ result corresponds to the change when taking $\mu_{0} / 2\left(2 \mu_{0}\right)$ as renormalization scale. The lower $\overline{\mathrm{MS}}$ uncertainty for BP $2_{2 A}$ is not shown, since the RGEs (3.5) do not allow for a stable solution, see also figure 9 of appendix B.

\begin{tabular}{|c|c|c|c|c|c|c|}
\hline $\mathrm{BP}$ & $\frac{\hat{\sigma}^{\mathrm{LO}}}{\mathrm{fb}}$ & $\bar{K}_{\mathrm{EW}}^{\mathrm{OS}}$ & $\bar{K}_{\mathrm{EW}}^{p^{*}}$ & $\bar{K}_{\mathrm{EW}}^{\text {proc1 }}$ & $\bar{K}_{\mathrm{EW}}^{\text {proc2 }}$ & $\bar{K}_{\mathrm{EW}}^{\overline{\mathrm{MS}}}$ \\
\hline $2_{1 A}$ & 25.737 & $\begin{array}{c}0.486 \\
(0.360)\end{array}$ & $\begin{array}{c}0.492 \\
(0.369)\end{array}$ & $\begin{array}{c}0.426 \\
(0.276)\end{array}$ & $\begin{array}{c}0.533 \\
(0.420)\end{array}$ & $\begin{array}{c}0.655_{-0.451}^{+0.197} \\
\left(0.590_{-0.733}^{+0.229}\right)\end{array}$ \\
\hline $2_{1 B}$ & 25.737 & $\begin{array}{c}0.177 \\
(-0.765)\end{array}$ & $\begin{array}{c}0.178 \\
(-0.756)\end{array}$ & $\begin{array}{c}0.166 \\
(-0.823)\end{array}$ & $\begin{array}{c}0.183 \\
(-0.773)\end{array}$ & $\begin{array}{c}0.257_{-0.060}^{-0.033} \\
\left(-0.387_{-1.336}^{-0.181}\right)\end{array}$ \\
\hline $2_{1 C}$ & 69.019 & $\begin{array}{c}0.958 \\
(0.939)\end{array}$ & $\begin{array}{c}0.950 \\
(0.931)\end{array}$ & $\begin{array}{c}0.822 \\
(0.780)\end{array}$ & $\begin{array}{c}0.904 \\
(0.879)\end{array}$ & $\begin{array}{c}0.822_{-0.088}^{+0.176} \\
\left(0.793_{-0.097}^{+0.181}\right)\end{array}$ \\
\hline $2_{1 D}$ & 69.019 & $\begin{array}{c}0.854 \\
(0.845)\end{array}$ & $\begin{array}{c}0.840 \\
(0.831)\end{array}$ & $\begin{array}{c}0.803 \\
(0.788)\end{array}$ & $\begin{array}{c}0.848 \\
(0.816)\end{array}$ & $\begin{array}{c}0.693_{-0.095}^{+0.244} \\
\left(0.662_{-0.117}^{+0.269}\right)\end{array}$ \\
\hline $3_{A 1}$ & 6.205 & $\begin{array}{c}0.581 \\
(0.305)\end{array}$ & $\begin{array}{c}0.580 \\
(0.303)\end{array}$ & $\begin{array}{c}0.486 \\
(0.210)\end{array}$ & $\begin{array}{c}0.550 \\
(0.245)\end{array}$ & $\begin{array}{c}0.336_{-0.100}^{+0.667} \\
\left(-0.150_{-0.121}^{-0.223}\right)\end{array}$ \\
\hline
\end{tabular}

Table 5. The NLO electroweak K-factors for the process $g+g \rightarrow H_{\mathrm{h}}$ for the $2 \mathrm{HDM}$ benchmark points (BP) in the alignment limit are shown. They are defined in table 1. Both $\bar{K}_{\mathrm{EW}}^{\mathrm{NLO}}$ (first value) and $K_{\mathrm{EW}}^{\mathrm{NLO}}$ (in parentheses) are shown. The renormalization scale has been set to $\mu_{0}$ as defined in eq. (3.4) for the $\overline{\mathrm{MS}}$ scheme. The lower (upper) value of the $\overline{\mathrm{MS}}$ result corresponds to the change when taking $\mu_{0} / 2\left(2 \mu_{0}\right)$ as renormalization scale. 
small and in particular comparable in size to the NLO contribution. Then, further terms of the perturbative expansion of the cross section are required

$$
\begin{aligned}
\left|A_{H_{\mathrm{h}}}^{\mathrm{NNLOEW}}\right|^{2}= & \left|A_{H_{\mathrm{h}}}^{(1)}+A_{H_{\mathrm{h}}}^{(2)}+A_{H_{\mathrm{h}}}^{(3)}\right|^{2} \\
= & \left|A_{H_{\mathrm{h}}}^{(1)}\right|^{2}+A_{H_{\mathrm{h}}}^{(1)} A_{H_{\mathrm{h}}}^{(2) *}+A_{H_{\mathrm{h}}}^{(1) *} A_{H_{\mathrm{h}}}^{(2)}+\left|A_{H_{\mathrm{h}}}^{(2)}\right|^{2} \\
& +A_{H_{\mathrm{h}}}^{(1)} A_{H_{\mathrm{h}}}^{(3) *}+A_{H_{\mathrm{h}}}^{(1) *} A_{H_{\mathrm{h}}}^{(3)}+\mathcal{O}\left(\left(G_{F} M_{W}^{2}\right)^{3}\right),
\end{aligned}
$$

where $A_{H_{\mathrm{h}}}^{(3)}$ is the three-loop amplitude. In eq. (5.2), $\left|A_{H_{\mathrm{h}}}^{(1)}\right|^{2}$ is the LO cross section up to a global factor. Likewise, $A_{H_{\mathrm{h}}}^{(1)} A_{H_{\mathrm{h}}}^{(2) *}+A_{H_{\mathrm{h}}}^{(1) *} A_{H_{\mathrm{h}}}^{(2)}$ corresponds to the NLO contribution, while $\left|A_{H_{\mathrm{h}}}^{(2)}\right|^{2}$ and $A_{H_{\mathrm{h}}}^{(1)} A_{H_{\mathrm{h}}}^{(3) *}+A_{H_{\mathrm{h}}}^{(1) *} A_{H_{\mathrm{h}}}^{(3)}$ enter at NNLO. If $\left|A_{H_{\mathrm{h}}}^{(1)}\right|^{2}$ is very small, $\left|A_{H_{\mathrm{h}}}^{(2)}\right|^{2}$ can be of the same size as the NLO contribution, while $A_{H_{\mathrm{h}}}^{(1)} A_{H_{\mathrm{h}}}^{(3) *}+A_{H_{\mathrm{h}}}^{(1) *} A_{H_{\mathrm{h}}}^{(3)}$ can be expected to be small again. Therefore, $\left|A_{H_{\mathrm{h}}}^{(2)}\right|^{2}$ should not be neglected. Furthermore, the imaginary part of the LO amplitude can be similar in magnitude or even larger than the real part when the heavy Higgs-boson mass becomes larger than twice the top-quark mass. This can lead to cancellations between real and imaginary contributions in the NLO term.

In both cases, there can be a considerable difference between $K_{\mathrm{EW}}^{\mathrm{NLO}}$ and $\bar{K}_{\mathrm{EW}}^{\mathrm{NLO}}$ as defined in eqs. (4.6) and (4.7). Since $\bar{K}_{\mathrm{EW}}^{\mathrm{NLO}}$ contains the additional contribution, it seems to be more appropriate to quantify the electroweak corrections to heavy Higgs-boson production. In order to point out BPs where this difference occurs, the $K_{\mathrm{EW}}^{\mathrm{NLO}}$ are shown in parentheses in tables 5 and 6 even though they can take an unphysical, negative value for some benchmark points, e.g. for $2_{1 B}$ and $3_{B 1}$.

Different from the sister process $g+g \rightarrow H_{1}$, many K-factors in tables 5 and 6 are far from one. This is at least partly due to the different LO couplings between the $t-H_{l}-t$ and $t-H_{h}-t$ interactions. While the $\left|c_{H_{1}}^{2 \mathrm{HDM}}\right|^{2}$ in tables 1 and 2 are generally one or close to one, the $\left|c_{H_{\mathrm{h}}}^{2 \mathrm{HDM}}\right|^{2}$ are at most 0.57 . In addition, the decoupling of the new Higgs sector from the SM can play a role for BP a-1 and $2_{2 A}$. While decoupling imposes the restriction that SM processes may not receive large corrections from the new sector, this is not the case for non-SM processes like heavy Higgs-boson production.

In the following, we have a closer look at the benchmark points $2_{1 B}, 2_{2 A}$ and $3_{B 1}$, which have large electroweak NLO corrections and a large difference between $\bar{K}_{\mathrm{EW}}^{\mathrm{NLO}}$ and $K_{\mathrm{EW}}^{\mathrm{NLO}}$ for heavy Higgs-boson production. Benchmark point $2_{1 B}$ does not only show very large corrections of more than $-80 \%$, but also the difference between $\bar{K}_{\mathrm{EW}}^{\mathrm{NLO}}$ and $K_{\mathrm{EW}}^{\mathrm{NLO}}$ is of the same order of magnitude. Hence, $\left|A_{H_{\mathrm{h}}}^{(1)}\right|$ and $\left|A_{H_{\mathrm{h}}}^{(2)}\right|$ must be similar in size, which may partially be due to the large value of $\left|\lambda_{i}^{\max }\right| /(4 \pi)=0.57$. The same is true for benchmark point $2_{2 A}$. It has a large value of $\left|\lambda_{i}^{\max }\right| /(4 \pi)=0.64$, and in addition, its LO cross section is quite small, such that the two-loop diagrams yield the true LO contribution. In order to verify whether the NNLO contribution is small in these scenarios, would, however, require the calculation of the three-loop contributions, which is not within reach in the near future.

The benchmark point $3_{B 1}$ has an even smaller LO cross section due to the almost vanishing coefficient $\left|c_{H_{\mathrm{h}}}^{2 \mathrm{HDM}}\right|^{2}=0.0003$. Therefore, the extremely large K-factor of more than 27 can be understood, since the true LO contribution is given by the two-loop diagrams. However, since the coefficients in front of the counterterms of the mixing angles are 


\begin{tabular}{|c|c|c|c|c|c|c|}
\hline $\mathrm{BP}$ & $\frac{\hat{\sigma}^{\mathrm{LO}}}{\mathrm{fb}}$ & $\bar{K}_{\mathrm{EW}}^{\mathrm{OS}}$ & $\bar{K}_{\mathrm{EW}}^{p^{*}}$ & $\bar{K}_{\mathrm{EW}}^{\text {proc1 }}$ & $\bar{K}_{\mathrm{EW}}^{\text {proc2 }}$ & $\bar{K}_{\mathrm{EW}}^{\overline{\mathrm{MS}}}$ \\
\hline $\mathrm{a}-1$ & 45.488 & $\begin{array}{c}1.145 \\
(1.031)\end{array}$ & $\begin{array}{c}1.161 \\
(1.048)\end{array}$ & $\begin{array}{c}1.121 \\
(1.011)\end{array}$ & $\begin{array}{c}1.084 \\
(0.997) \\
\end{array}$ & $\begin{array}{c}1.401_{-1.385}^{+0.253} \\
\left(1.270_{-2.114}^{+0.200}\right) \\
\end{array}$ \\
\hline b-1 & 10.767 & $\begin{array}{c}0.696 \\
(0.569)\end{array}$ & $\begin{array}{c}0.693 \\
(0.566)\end{array}$ & $\begin{array}{c}0.628 \\
(0.499)\end{array}$ & $\begin{array}{c}0.701 \\
(0.572)\end{array}$ & $\begin{array}{c}0.981_{-0.574}^{+0.154} \\
\left(0.898_{-0.713}^{+0.140}\right)\end{array}$ \\
\hline $2_{2 A}$ & 2.866 & $\begin{array}{c}7.504 \\
(2.798) \\
\end{array}$ & $\begin{array}{c}7.456 \\
(2.773)\end{array}$ & $\begin{array}{l}7.517 \\
(2.812)\end{array}$ & $\begin{array}{c}6.989 \\
(2.738)\end{array}$ & $\begin{array}{c}4.030+0.758 \\
- \\
\left(-0.273^{+3.301}\right)\end{array}$ \\
\hline $3_{B 1}$ & 0.019 & $\begin{array}{c}27.00 \\
(-2.714)\end{array}$ & $\begin{array}{c}27.09 \\
(-2.821)\end{array}$ & $\begin{array}{c}326.3 \\
(-24.62)\end{array}$ & $\begin{array}{c}37.35 \\
(-2.739)\end{array}$ & $\begin{array}{r}286.5_{-285.5}^{-284.9} \\
\left(-33.26_{+30.66}^{+34.70}\right)\end{array}$ \\
\hline $3_{B 2}$ & 2.586 & $\begin{array}{c}1.019 \\
(0.933)\end{array}$ & $\begin{array}{l}1.031 \\
(0.945)\end{array}$ & $\begin{array}{c}1.005 \\
(0.828)\end{array}$ & $\begin{array}{c}1.042 \\
(0.850)\end{array}$ & $\begin{array}{c}7.491_{+23.13}^{-6.210} \\
\left(4.443_{+5.347}^{-3.181}\right)\end{array}$ \\
\hline $4_{3}$ & 9.828 & $\begin{array}{c}0.945 \\
(0.887) \\
\end{array}$ & $\begin{array}{c}0.941 \\
(0.883)\end{array}$ & $\begin{array}{c}0.979 \\
(0.910)\end{array}$ & $\begin{array}{c}1.151 \\
(0.925)\end{array}$ & $\begin{array}{r}0.580{ }_{-0.171}^{+0.531} \\
\left(0.450_{-0.237}^{+0.574}\right) \\
\end{array}$ \\
\hline $4_{4}$ & 10.271 & $\begin{array}{c}1.028 \\
(0.977) \\
\end{array}$ & $\begin{array}{c}1.024 \\
(0.973)\end{array}$ & $\begin{array}{c}1.067 \\
(1.006) \\
\end{array}$ & $\begin{array}{c}1.216 \\
(1.032) \\
\end{array}$ & $\begin{array}{r}0.807_{-0.190}^{+0.322} \\
\left(0.740_{-0.224}^{+0.326}\right)\end{array}$ \\
\hline $4_{5}$ & 10.552 & $\begin{array}{c}0.794 \\
(0.782)\end{array}$ & $\begin{array}{c}0.799 \\
(0.788)\end{array}$ & $\begin{array}{c}0.844 \\
(0.838)\end{array}$ & $\begin{array}{c}0.749 \\
(0.591)\end{array}$ & $\begin{array}{r}1.096_{+1.155}^{-0.402} \\
\left(1.094_{+0.905}^{-0.429}\right)\end{array}$ \\
\hline
\end{tabular}

Table 6. The NLO electroweak K-factors for the process $g+g \rightarrow H_{\mathrm{h}}$ for the $2 \mathrm{HDM}$ benchmark points (BP) that are not in the alignment limit are shown. They are defined in table 2. The renormalization scale has been set to $\mu_{0}$ as defined in eq. (3.4) for the $\overline{\mathrm{MS}}$ scheme. Both $\bar{K}_{\mathrm{EW}}^{\mathrm{NLO}}$ (first value) and $K_{\mathrm{EW}}^{\mathrm{NLO}}$ (in parentheses) are shown. The lower (upper) value of the $\overline{\mathrm{MS}}$ result corresponds to the change when taking $\mu_{0} / 2\left(2 \mu_{0}\right)$ as renormalization scale. The lower $\overline{\mathrm{MS}}$ uncertainty for BP $2_{2 A}$ is not shown, since the RGEs (3.5) do not allow for a stable solution, see also figure 9 of appendix B.

large for this $\mathrm{BP}$ and the small LO does not factorize, we observe large differences between the results in different renormalization schemes. This leads to a large dependence on the choice of the renormalization scheme, and thus to large theoretical uncertainties on the production cross section. In particular, this shows that a small coupling of the heavy Higgs boson to top quarks does not automatically lead to a small cross section when higher order contributions are considered.

Finally, there are benchmark points that have moderate NLO corrections on the one hand, but a relatively large difference between $\bar{K}_{\mathrm{EW}}^{\mathrm{NLO}}$ and $K_{\mathrm{EW}}^{\mathrm{NLO}}$ on the other hand. We have investigated this difference further and found that it is caused by cancellations between the real and imaginary contributions in the term $A_{H_{\mathrm{h}}}^{(1)} A_{H_{\mathrm{h}}}^{(2) *}+A_{H_{\mathrm{h}}}^{(1 *)} A_{H_{\mathrm{h}}}^{(2)}$. Especially benchmark point a-1 exhibits this feature, but it can also be observed in BP $3_{B 2}$ and $4_{4}$.

As a measure of the scale dependence in the $\overline{\mathrm{MS}}$ scheme, we can look at the size of the coefficients of the scale-dependent logarithms in the percentage correction presented 
in tables 7 and 8 of appendix A. In general, they can become very small or even vanish for certain choices of the parameters. In particular, in the alignment limit with $M_{\mathrm{sb}}=0$, the scale dependent logarithmic term for $g+g \rightarrow H_{1}$ can be obtained from eq. (A.1) of appendix $\mathrm{A}$ and becomes very simple

$$
\delta_{\mathrm{EW}, M_{\mathrm{sb}}=0}^{\mathrm{NLO}, \text { dep. }}=\frac{3 G_{F} \sqrt{2} M_{H_{1}}^{2}}{8 \pi^{2} t_{\beta}^{2}\left(M_{H_{\mathrm{h}}}^{2}-M_{H_{1}}^{2}\right)} \ln \left(\frac{\mu^{2}}{M_{H_{1}}^{2}}\right)\left[\left(1-t_{\beta}^{2}\right) M_{H_{\mathrm{h}}}^{2}+2 m_{t}^{2}\right],
$$

which even vanishes for the special case $M_{H_{\mathrm{h}}}^{2}=2 m_{t}^{2} /\left(t_{\beta}^{2}-1\right)$. This scenario is almost realized for BP $2_{1 C}$ and $2_{1 D}$, and hence, these benchmark points exhibit only a small scale dependence in table 3 .

In addition, we observe that the $\overline{\mathrm{MS}}$ corrections for light Higgs-boson production are usually moderate, while for heavy Higgs-boson production, this scheme often leads to Kfactors far from one in addition to a very strong scale dependence. This can, again, partly be traced back to small LO couplings between the heavy Higgs boson and the top quarks.

Benchmark scenarios at $\boldsymbol{M}^{*}=\mathbf{7 0 0} \mathrm{GeV}$. Next, we consider light and heavy Higgsboson production in two benchmark scenarios at a moderately heavy mass scale $M^{*}$

$$
M_{H_{\mathrm{a}}}=M_{H_{\mathrm{c}}}=M_{\mathrm{sb}}=M^{*}=700 \mathrm{GeV}, \quad t_{\beta}=2, \quad M_{H_{\mathrm{h}}}=600 \ldots 800 \mathrm{GeV} .
$$

The first scenario uses the alignment limit and the second scenario sets $c_{\alpha \beta}=0.03$. As we see in figure 5 , both scenarios fulfill the perturbativity restriction $\left|\lambda_{i}\right| /(4 \pi)<1$ ( $i=$ $1, \ldots, 5)$. In addition, they respect the experimental constraints considered in refs. [7, 69]. In both scenarios we vary the heavy, scalar Higgs-boson mass between 600 and $800 \mathrm{GeV}$. The size of the couplings $\lambda_{i}$ is more sensitive to these variations compared to variations in the other Higgs-boson masses $M_{H_{\mathrm{a}}}, M_{H_{\mathrm{c}}}$ (compare figure 5 to figure 8 in appendix B). For that reason we will present the NLO corrections to Higgs-boson production and also the scale dependence in the $\overline{\mathrm{MS}}$ renormalization scheme as a function of the heavy Higgs-boson mass in the following.

Figure 6 shows the K-factor of the NLO electroweak corrections for the process $g+g \rightarrow$ $H_{\mathrm{l}}$ in the $2 \mathrm{HDM}$ as a function of the heavy, neutral Higgs-boson mass for the different renormalization schemes of the mixing angles $\alpha$ and $\beta$. We consider the OS, $p^{*}$, two processdependent and the $\overline{\mathrm{MS}}$ scheme. The grey shaded band shows the region where at least one of the couplings $\left|\lambda_{i}\right| /(4 \pi)$ of eq. (2.2) becomes larger than 0.5 . The region of variation of the corresponding $\left|\lambda_{i}\right| /(4 \pi)$ values is displayed in figure 5 . These values are not valid for the $\overline{\mathrm{MS}}$ scheme, where they adopt different values depending on the choice of the renormalization scale. As a result of this, the region of perturbativity generally looks different for the $\overline{\mathrm{MS}}$ renormalized results. In the $\overline{\mathrm{MS}}$ scheme the corrections are renormalization-scale dependent and we choose the central renormalization scale $\mu_{0}$ according to eq. (3.4). In order to estimate the impact of the scale dependence on the size of the NLO EW corrections, we vary the scale between $\mu_{0} / 2$ and $2 \mu_{0}$. A more detailed analysis is discussed in appendix C. In the alignment limit presented in the first plot of figure 6 , the different renormalization schemes agree quite well for $\left|\lambda_{i}\right| /(4 \pi)<0.5$. The curves for $\overline{\mathrm{MS}}$ renormalization (blue) and 


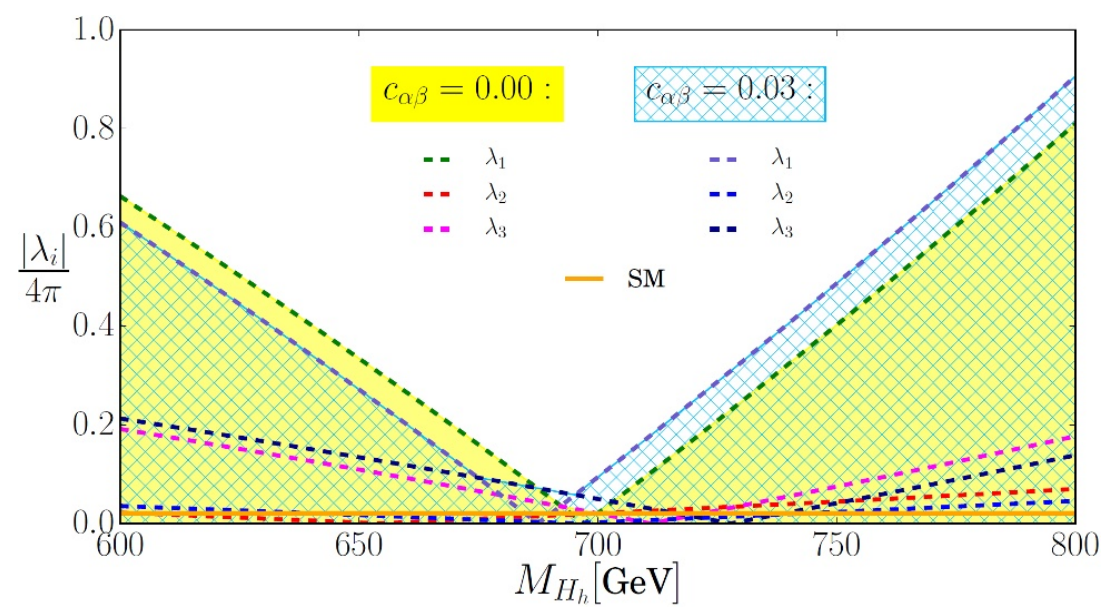

Figure 5. The range of $\left|\lambda_{i}\right| /(4 \pi)$ for our benchmark scenarios compared to the SM value $\lambda^{\mathrm{SM}} /(4 \pi)=0.02$ (solid, orange line). For the two different values of $c_{\alpha \beta}$ the individual curves for those $\left|\lambda_{i}\right| /(4 \pi)$ that are different from zero are also shown (dashed lines). The explicit formulae for the parameters $\lambda_{i}$ are given in eqs. (B.1)-(B.5) of appendix B. The values shown here are for the non- $\overline{\mathrm{MS}}$ schemes and thus no running is taken into account.

the renormalization scheme proc1 (purple, dashed-dotted) show the biggest deviation from the SM, especially when entering the non-perturbative region. The corrections of the OS, $p^{*}$ and proc2 renormalization schemes are very close to each other for these scenarios, even for large $\lambda_{i}$. For $c_{\alpha \beta}=0.03$ presented in the second plot of figure 6 , the behaviour of the OS, $p^{*}$, proc1 and proc 2 scheme is very similar to the case of the alignment limit (upper plot), while the $\overline{\mathrm{MS}}$ results show a much larger scale variation.

Figure 7 shows the K-factor of the NLO electroweak corrections in different renormalization schemes for the process $g+g \rightarrow H_{\mathrm{h}}$ in the 2HDM as a function of the heavy, neutral Higgs-boson mass for the same scenarios as in figure 6. Again, as for the benchmark points, we use $\bar{K}_{\mathrm{EW}}^{\mathrm{NLO}}$ as the K-factor for heavy Higgs-boson production. First of all, we can expect the NLO corrections for heavy Higgs-boson production to be larger: the LO contribution is suppressed since for $t_{\beta}=2$ and close to the alignment limit, the coefficient $c_{H_{\mathrm{h}}}^{2 \mathrm{HDM}} \approx-0.5$ is considerably smaller than $c_{H_{1}}^{2 \mathrm{HDM}} \approx 1$. In addition, the LO depends on the heavy Higgsboson mass $M_{H_{\mathrm{h}}}$, which is not fixed in the scenario under consideration. In general, we can see that for $\left|\lambda_{i}^{\max }\right| /(4 \pi)<0.5$, the K-factors do no longer lie roughly between 0.99 and 1.12 as for light Higgs-boson production, but now we observe a larger range of K-factors between 0.4 and values larger than 1.8. Just as for light Higgs-boson production, the proc1 scheme differs considerably from the other renormalization schemes for large $M_{H_{\mathrm{h}}}$. The behaviour of the results for the $\overline{\mathrm{MS}}$ renormalization seems to be more similar to the other schemes when compared with the light Higgs-boson production, even though there are sizable numerical differences in some regions. However, this scheme strongly depends on the definition of the running procedure requiring a more detailed analysis, which is performed in appendix $\mathrm{C}$.

As expected, due to cancellations among the finite counterterms, the OS and $p^{*}$ scheme lead to small perturbative corrections. The differences between these two schemes may be 

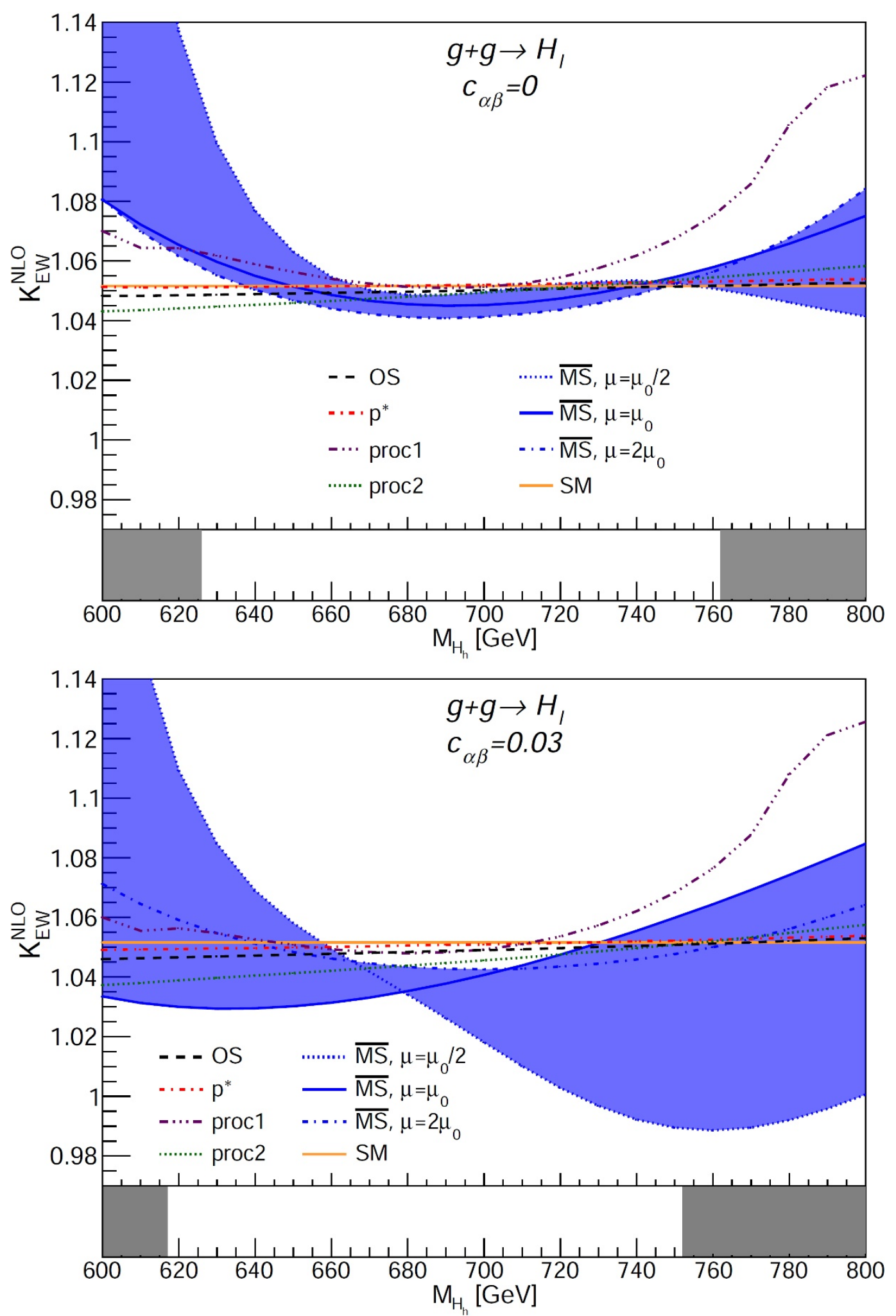

Figure 6. Percentage correction of the process $g+g \rightarrow H_{1}$ in various schemes. The grey shaded band denotes the region where at least one of the couplings $\left|\lambda_{i}\right| /(4 \pi)$ becomes larger than 0.5 and where one slowly starts to enter in the non-perturbative regime. 

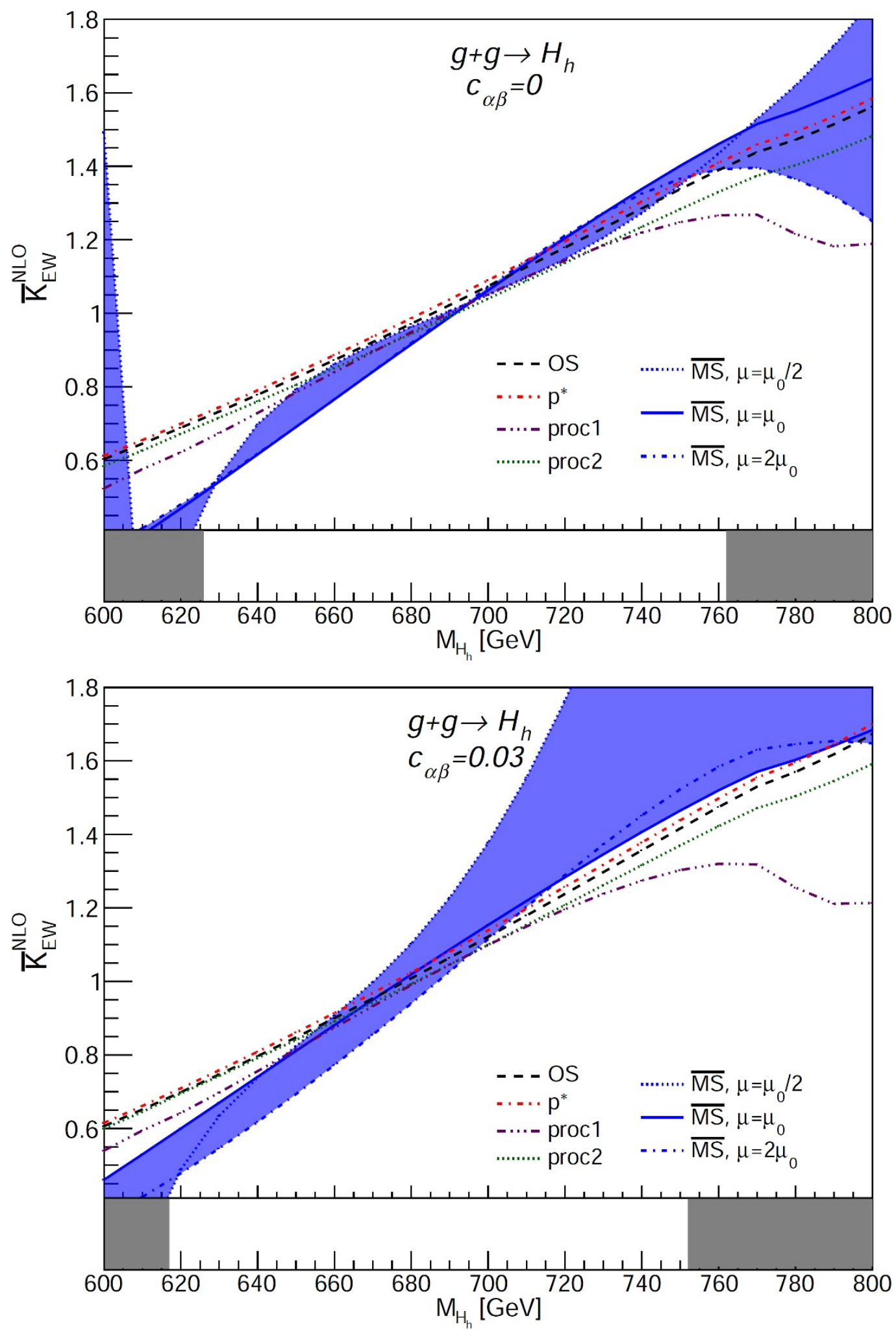

Figure 7. Percentage correction of the process $g+g \rightarrow H_{\mathrm{h}}$ in various schemes. The grey shaded band denotes the region where at least one of the couplings $\left|\lambda_{i}\right| /(4 \pi)$ becomes larger than 0.5 and where one slowly starts to enter in the non-perturbative regime. 
too small to give an estimate of missing higher-order uncertainties. As long as $M_{H_{\mathrm{h}}}$ does not become too large the two process-dependent schemes also perform very well for the analyzed scenarios, both for light as well as for heavy Higgs-boson production.

\section{Summary and conclusion}

We have computed the two-loop electroweak corrections to the production of a light and a heavy neutral, scalar Higgs boson through gluon fusion in the 2HDM. We have renormalized the new Higgs-boson masses in the on-shell scheme and provide the electroweak percentage correction in different renormalization schemes for the mixing angles $\alpha$ and $\beta$ for these two processes. In particular, for the mixing angles we have employed the on-shell, $p^{*}$, $\overline{\mathrm{MS}}$ and two process dependent schemes. We can determine the next-to-leading order, electroweak percentage correction for essentially any scenario of the new mass parameters and the mixing angles of the CP-conserving 2HDM. In particular, we have computed the two-loop electroweak corrections for benchmark points collected by the LHC Higgs cross section working group as well as for individual other example scenarios. For Higgsboson production through gluon fusion, the on-shell scheme performs well for all chosen scenarios. The $\overline{\mathrm{MS}}$ scheme can suffer from a large scale dependence and can in general not provide reliable predictions for all scenarios. For the production of the light Higgs-boson, the electroweak corrections are always moderate in size, i.e. they are mostly around $5 \%$ compared to the LO production cross section for the chosen benchmark points, while for the production of a heavy Higgs-boson, the electroweak corrections strongly vary depending on the details of the selected scenario. We have solved new technical challenges, which was required to accomplish this calculation. Our results are also directly applicable to determine the electroweak percentage corrections for the partial decay widths of the light and heavy neutral, scalar Higgs-boson decay into two gluons within the 2HDM.

\section{Acknowledgments}

We would like to thank Ansgar Denner and Jean-Nicolas Lang for valuable discussions and numerical comparisons. The work of L.J. and C.S. was supported by the Deutsche Forschungsgemeinschaft (DFG) under contract STU 615/1-1. The work of S.U. was supported in part by the European Commission through the "HiggsTools" Initial Training Network PITN-GA-2012-316704. The computations were performed with the help of the High Performance Computing (HPC) cluster of the University of Würzburg, DFG project number 327497565. The Feynman diagrams were drawn with the program Axodraw [74, 75].

\section{A Scale dependence of the percentage correction in the $\overline{\mathrm{MS}}$ scheme with- out running of $c_{\alpha \beta}$ and $t_{\beta}$}

The NLO EW percentage corrections $\delta_{\mathrm{EW}}^{\mathrm{NLO}}$ to the LO partonic cross section are defined through $\hat{\sigma}^{\mathrm{NLO}}=\hat{\sigma}^{\mathrm{LO}}\left(1+\delta_{\mathrm{EW}}^{\mathrm{NLO}}\right)$. The scale dependence of the percentage correction in the 
$\overline{\mathrm{MS}}$ scheme for the process $g+g \rightarrow H_{1}$ in the alignment limit $\left(c_{\alpha \beta}=0\right)$ has already been presented in ref. [27]. It reads

$$
\begin{aligned}
\delta_{\mathrm{EW}}^{\mathrm{NLO}, \mu-\mathrm{dep} .}= & \frac{G_{F} \sqrt{2}}{8 \pi^{2} t_{\beta}^{2} M_{H_{\mathrm{h}}}^{2}\left(M_{H_{\mathrm{h}}}^{2}-M_{H_{1}}^{2}\right)} \ln \left(\frac{\mu^{2}}{M_{H_{1}}^{2}}\right) \\
& \times\left\{\left(1-t_{\beta}^{2}\right)\left(M_{H_{\mathrm{h}}}^{2}-M_{\mathrm{sb}}^{2}\right)\left[3 M_{H_{\mathrm{h}}}^{2} M_{H_{1}}^{2}+M_{\mathrm{sb}}^{2}\left(M_{H_{\mathrm{a}}}^{2}+2 M_{H_{\mathrm{c}}}^{2}-3 M_{H_{\mathrm{h}}}^{2}\right)\right]\right. \\
& \left.+6 m_{t}^{2}\left(M_{H_{\mathrm{h}}}^{2} M_{H_{1}}^{2}-4 M_{\mathrm{sb}}^{2} m_{t}^{2}\right)\right\} .
\end{aligned}
$$

The corresponding scale dependence for the process $g+g \rightarrow H_{\mathrm{h}}$ in the alignment limit $\left(c_{\alpha \beta}=0\right)$ is given by

$$
\begin{aligned}
\delta_{\mathrm{EW}}^{\mathrm{NLO}, \mu \text {-dep. }}= & \frac{G_{F} \sqrt{2}}{16 \pi^{2} t_{\beta}^{2} M_{H_{\mathrm{h}}}^{2}\left(M_{H_{\mathrm{h}}}^{2}-M_{H_{1}}^{2}\right)} \ln \left(\frac{\mu^{2}}{M_{H_{1}}^{2}}\right) \\
\times & \left\{( 1 - t _ { \beta } ^ { 2 } ) ( M _ { \mathrm { sb } } ^ { 2 } - M _ { H _ { \mathrm { h } } } ^ { 2 } ) \left[\left(M_{H_{\mathrm{a}}}^{2}+2 M_{H_{\mathrm{c}}}^{2}\right)\left[\left(1+t_{\beta}^{2}\right)\left(M_{H_{\mathrm{h}}}^{2}-M_{H_{1}}^{2}\right)+2 t_{\beta}^{2} M_{\mathrm{sb}}^{2}\right]\right.\right. \\
& \left.+3 M_{H_{\mathrm{h}}}^{2}\left[M_{H_{\mathrm{h}}}^{2}-M_{H_{1}}^{2}+t_{\beta}^{2}\left(M_{H_{\mathrm{h}}}^{2}+M_{H_{1}}^{2}-2 M_{\mathrm{sb}}^{2}\right)\right]\right] \\
& -6 m_{t}^{2} M_{H_{\mathrm{h}}}^{2}\left[M_{H_{\mathrm{h}}}^{2}-M_{H_{1}}^{2}+t_{\beta}^{2}\left(M_{H_{\mathrm{h}}}^{2}+M_{H_{1}}^{2}\right)\right] \\
& \left.+24 m_{t}^{4}\left[\left(1+t_{\beta}^{2}\right)\left(M_{H_{\mathrm{h}}}^{2}-M_{H_{1}}^{2}\right)+2 t_{\beta}^{2} M_{\mathrm{sb}}^{2}\right]\right\} .
\end{aligned}
$$

The scale dependence of the process $g+g \rightarrow H_{\mathrm{h}}$ in the anti-alignment limit $\left(s_{\alpha \beta}=0\right)$ can be obtained from eq. (A.1) by interchanging the light and heavy Higgs-boson masses, i.e. $M_{H_{1}} \leftrightarrow M_{H_{\mathrm{h}}}$. Likewise one can obtain the scale dependence of the process $g+g \rightarrow H_{\mathrm{l}}$ in the anti-alignment limit $\left(s_{\alpha \beta}=0\right)$ from eq. (A.2) by again interchanging the light and heavy Higgs-boson masses. The limit of two equal neutral, scalar Higgs-boson masses, $M_{H_{\mathrm{h}}} \equiv M_{H_{1}}$, does not exist in all the above cases due to the denominator structure of eqs. (A.1) and (A.2).

In order to study and judge the magnitude of the scale dependence, we compare the size of the coefficient of the scale dependent logarithm, which we define by

$$
\delta_{\mathrm{EW}}^{\mathrm{NLO}, \mu \text {-dep. }}(x)=d(x) \ln \left(\frac{\mu^{2}}{M_{H_{1}}^{2}}\right), \quad \text { with } \quad x=g+g \rightarrow H_{l} \quad \text { or } \quad x=g+g \rightarrow H_{h} .
$$

In particular for the BPs in the alignment limit, the coefficient can easily be obtained by eqs. (A.1) and (A.2). The explicit values of the coefficients are shown in tables 7 and 8 . The size of the coefficients reflects directly the magnitude of the scale dependence in the $\overline{\mathrm{MS}}$ scheme without taking into account the running of the mixing angles $c_{\alpha \beta}$ and $t_{\beta}$. Coefficients whose absolute value is larger than one, like for example for the BPs $2_{2 A}, 3_{B 1}$ and $3_{B 2}$ for the process $g+g \rightarrow H_{h}$, exhibit a large scale dependence and give rise to large corrections. Small coefficients, like for example for the BPs $2_{1 C}, 2_{1 D}, 2_{2 A}$ and $3_{B 2}$ for the process $g+g \rightarrow H_{l}$, exhibit a very small scale dependence. 


\begin{tabular}{|c|c|c|c|c|c|}
\hline $\mathrm{BP}$ & $2_{1 A}$ & $2_{1 B}$ & $2_{1 C}$ & $2_{1 D}$ & $3_{A 1}$ \\
\hline$d\left(g+g \rightarrow H_{l}\right)$ & -0.071 & -0.072 & -0.004 & -0.004 & -0.058 \\
\hline$d\left(g+g \rightarrow H_{h}\right)$ & 0.215 & 0.218 & 0.122 & 0.155 & 0.864 \\
\hline
\end{tabular}

Table 7. The coefficient in front of the scale dependent logarithm of the percentage correction is shown for the benchmark points that are in the alignment limit $\left(c_{\alpha \beta}=0\right)$.

\begin{tabular}{|c|c|c|c|c|c|c|c|c|}
\hline BP & a-1 & b-1 & $2_{2 A}$ & $3_{B 1}$ & $3_{B 2}$ & $4_{3}$ & $4_{4}$ & $4_{5}$ \\
\hline$d\left(g+g \rightarrow H_{l}\right)$ & -0.183 & -0.045 & $4 \cdot 10^{-5}$ & -0.029 & 0.003 & -0.015 & 0.003 & 0.072 \\
\hline$d\left(g+g \rightarrow H_{h}\right)$ & 0.562 & 0.283 & -5.672 & 15.917 & -1.507 & 0.353 & 0.201 & -0.331 \\
\hline
\end{tabular}

Table 8. The coefficient in front of the scale dependent logarithm of the percentage correction is shown for the benchmark points that are not in the alignment limit $\left(c_{\alpha \beta} \neq 0\right)$.

\section{B Behaviour of the coupling constants $\lambda_{i}$}

The parameters $\lambda_{i}(i=1, \ldots, 5)$ of the Higgs potential in eq. (2.2) depend on trigonometric functions of the mixing angles, i.e. $t_{\beta}$ and $c_{\alpha \beta}$ as well as on the masses of the Higgs-bosons and the soft-breaking scale. The individual parameters read

$$
\begin{aligned}
& \lambda_{1}=\frac{g^{2}}{4 M_{W}^{2}}\left[\left(c_{\alpha \beta}-s_{\alpha \beta} t_{\beta}\right)^{2} M_{H_{\mathrm{h}}}^{2}+\left(s_{\alpha \beta}+c_{\alpha \beta} t_{\beta}\right)^{2} M_{H_{1}}^{2}-t_{\beta}^{2} M_{\mathrm{sb}}^{2}\right], \\
& \lambda_{2}=\frac{g^{2}}{4 M_{W}^{2} t_{\beta}^{2}}\left[\left(s_{\alpha \beta}+c_{\alpha \beta} t_{\beta}\right)^{2} M_{H_{\mathrm{h}}}^{2}+\left(c_{\alpha \beta}-s_{\alpha \beta} t_{\beta}\right)^{2} M_{H_{1}}^{2}-M_{\mathrm{sb}}^{2}\right], \\
& \lambda_{3}=\frac{g^{2}}{4 M_{W}^{2} t_{\beta}}\left[\left(s_{\alpha \beta}+c_{\alpha \beta} t_{\beta}\right)\left(c_{\alpha \beta}-s_{\alpha \beta} t_{\beta}\right)\left(M_{H_{\mathrm{h}}}^{2}-M_{H_{1}}^{2}\right)+t_{\beta}\left(2 M_{H_{\mathrm{c}}}^{2}-M_{\mathrm{sb}}^{2}\right)\right], \\
& \lambda_{4}=\frac{g^{2}}{4 M_{W}^{2}}\left[M_{\mathrm{sb}}^{2}-2 M_{H_{\mathrm{c}}}^{2}+M_{H_{\mathrm{a}}}^{2}\right], \\
& \lambda_{5}=\frac{g^{2}}{4 M_{W}^{2}}\left[M_{\mathrm{sb}}^{2}-M_{H_{\mathrm{a}}}^{2}\right],
\end{aligned}
$$

with the coupling $g$ and the $W$-boson mass $M_{W}$.

Figure 8 shows the range of the parameters $\left|\lambda_{i}\right| /(4 \pi)$ of the potential for the alternative scenarios discussed in section 5, where we have fixed the values of the mixing angles to $t_{\beta}=2$ and $c_{\alpha \beta}=0$ or $c_{\alpha \beta}=0.03$. All new heavy mass scales are set to the same value of $700 \mathrm{GeV}$, except for one Higgs-boson mass, which is varied between 600 and $800 \mathrm{GeV}$. In the two plots of figure 8 the parameters $\left|\lambda_{i}\right| /(4 \pi)$ are shown as a function of $M_{H_{\mathrm{a}}}$ and $M_{H_{\mathrm{c}}}$, while the plot as a function of $M_{H_{\mathrm{h}}}$ is given in figure 5 of section 5 . The size of the couplings is less sensitive to a variation of $M_{H_{\mathrm{a}}}$ and $M_{H_{\mathrm{c}}}$ than to a variation of $M_{H_{\mathrm{h}}}$ as shown in figure 5 .

In figure 9 we show a bar chart, where we illustrate the maximal size $\left|\lambda_{i}^{\max }\right| /(4 \pi)$ of the couplings of the Higgs-boson potential of eq. (2.2) for an $\overline{\mathrm{MS}}$ renormalization of the mixing angles for the BPs of tables 1 and 2. The horizontal, dashed line shows the location of the value $\left|\lambda_{i}^{\max }\right| /(4 \pi)=0.5$. The values $\left|\lambda_{i}^{\max }\right| /(4 \pi)$ are determined with eqs. (B.1)-(B.5) of 

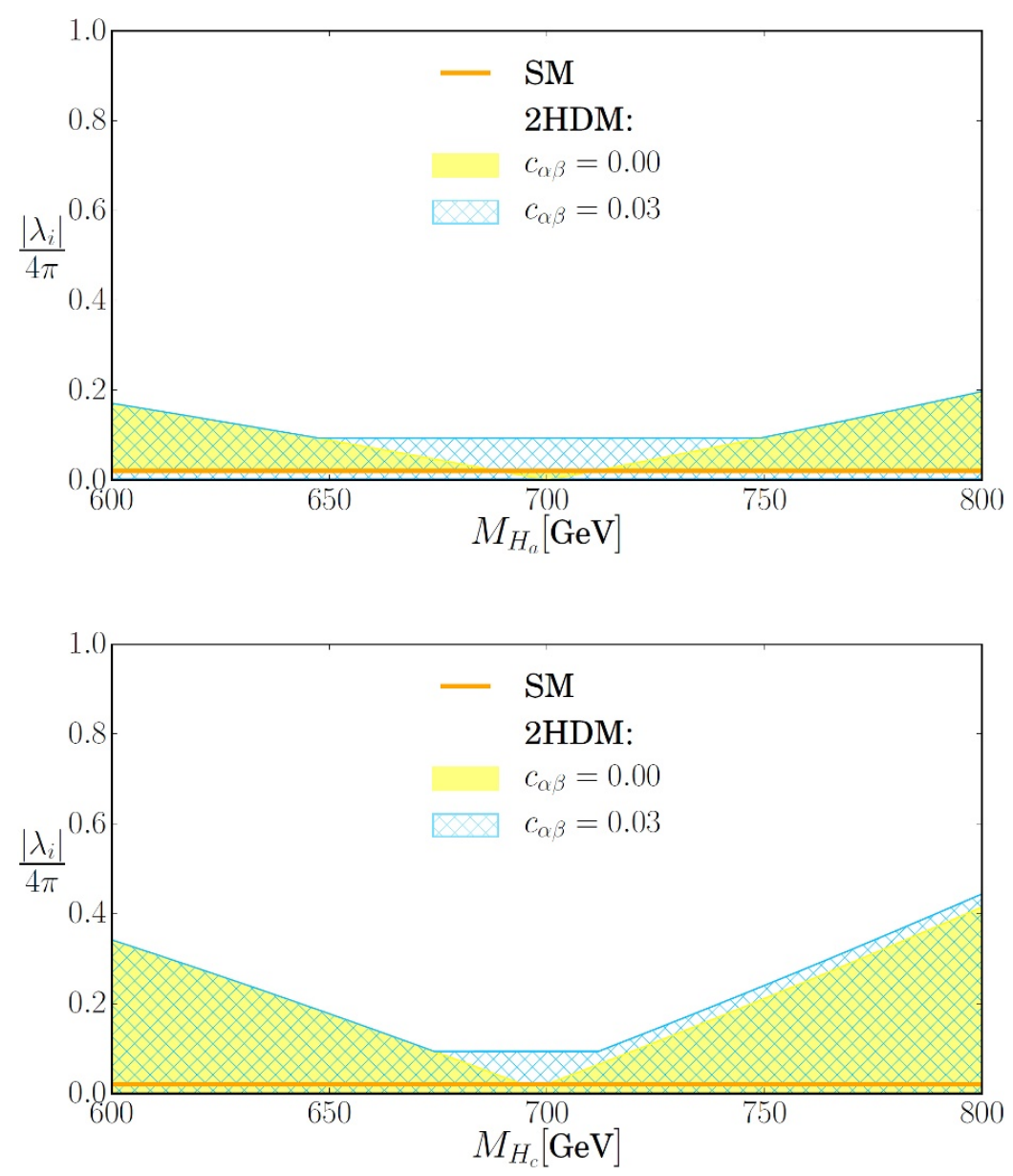

Figure 8. The range between the minimal and maximal value of the modulus of the parameters $\lambda_{i} /(4 \pi)$ as a function of one of the new Higgs-boson masses is shown. All other new Higgs-boson mass scales are kept fixed at $700 \mathrm{GeV}$. The range is also compared to the SM value $\lambda^{\mathrm{SM}} /(4 \pi)=0.02$ (solid, orange line). The values shown here are for the non- $\overline{\mathrm{MS}}$ schemes and thus no running is taken into account.

appendix $\mathrm{A}$ and are considered to be defined at the scale $\mu_{d}=\mu_{0}$ of eq. (3.4) here. The green central bars of $\left|\lambda_{i}^{\max }\right| /(4 \pi)$ coincide with the values given in tables 1 and 2 . In the $\overline{\mathrm{MS}}$ scheme these central values are then run with the help of the RGEs (3.5) to the scale $\mu_{0} / 2$ and $2 \mu_{0}$, and they are used to obtain the red and yellow bar, respectively.

\section{C $\overline{\mathrm{MS}}$ results in the $M^{*}$ benchmark scenarios}

In this appendix we give the results in the $\overline{\mathrm{MS}}$ renormalization scheme of the mixing angles $\alpha$ and $\beta$, for the K-factors of the benchmark scenarios at $M^{*}=700 \mathrm{GeV}$ described in section 5 . The mass parameters read

$$
M_{H_{\mathrm{a}}}=M_{H_{\mathrm{c}}}=M_{\mathrm{sb}}=M^{*}=700 \mathrm{GeV}, \quad M_{H_{\mathrm{h}}}=600, \ldots, 800 \mathrm{GeV},
$$




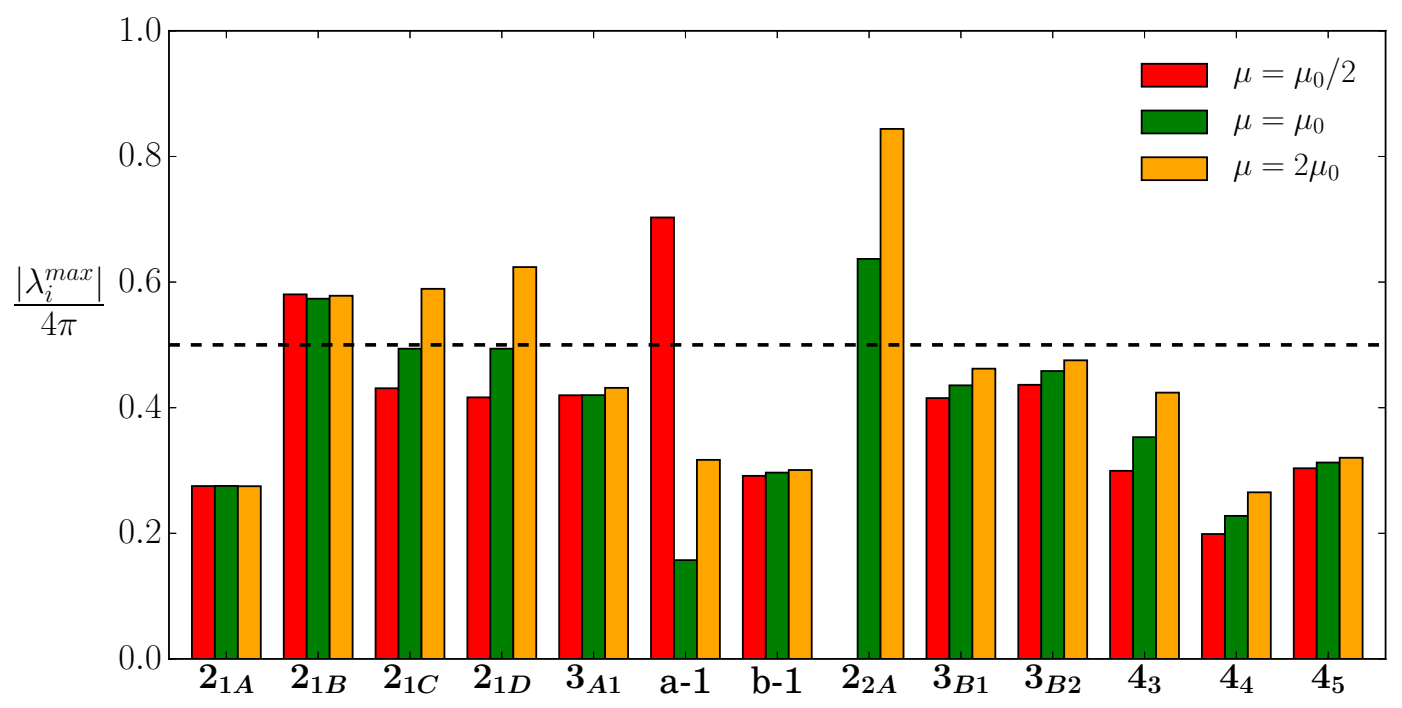

Figure 9. The running of the largest coupling constants $\left|\lambda_{i}^{\max }\right| /(4 \pi)$ is shown for all benchmark points of section 5. For the benchmark point $2_{2 A}$ the bar for $\mu=\mu_{0} / 2$ is not given, since the RGEs (3.5) do not allow for a stable solution.

and the mixing angles are

$$
t_{\beta}=2, \quad c_{\alpha \beta}=0 \quad \text { or } \quad c_{\alpha \beta}=0.03,
$$

see also eq. (5.4). In particular in figures 10 and 11 we compare the scale dependence of the EW corrections in three cases:

- The dependence on the renormalization scale $\mu$ enters just in the loop corrections. The running of the mixing angles $\alpha$ and $\beta$ is not taken into account (red bands).

- The dependence on the renormalization scale $\mu$ enters in the loop corrections as well as in the running of the mixing angles $\alpha$ and $\beta$, see eq. (3.5); the default scale is $\mu_{d}=\mu_{0}$, where the soft-breaking scale takes the value $M_{\mathrm{sb}}=700 \mathrm{GeV}$, and where the mixing angles $t_{\beta}$ and $c_{\alpha \beta}$ take the benchmark values $t_{\beta}=2$, and $c_{\alpha \beta}=0$ or $c_{\alpha \beta}=0.03$ (blue bands).

- The dependence on the renormalization scale $\mu$ enters in the loop corrections as well as in the running of the mixing angles $\alpha$ and $\beta$, see eq. (3.5); the default scale $\mu_{d}$ at which the soft-breaking scale takes the value $M_{\mathrm{sb}}=700 \mathrm{GeV}$ and at which the mixing angles $t_{\beta}$ and $c_{\alpha \beta}$ take the benchmark values $t_{\beta}=2$ and $c_{\alpha \beta}=0$ or $c_{\alpha \beta}=0.03$ is $\mu_{d}=\operatorname{vev}$ (yellow bands).

The bands in figures 10 and 11 are generated by considering the region of the parametric space between the three curves corresponding to the values $\mu_{0}, \mu_{0} / 2$ and $2 \mu_{0}$ for the renormalization scale, where $\mu_{0}$ is the typical scale of the processes under consideration as calculated in eq. (3.4). The curve of the on-shell renormalization scheme (dashed, black curve) is shown as a reference curve for the scale-independent schemes. For an $\overline{\mathrm{MS}}$ 

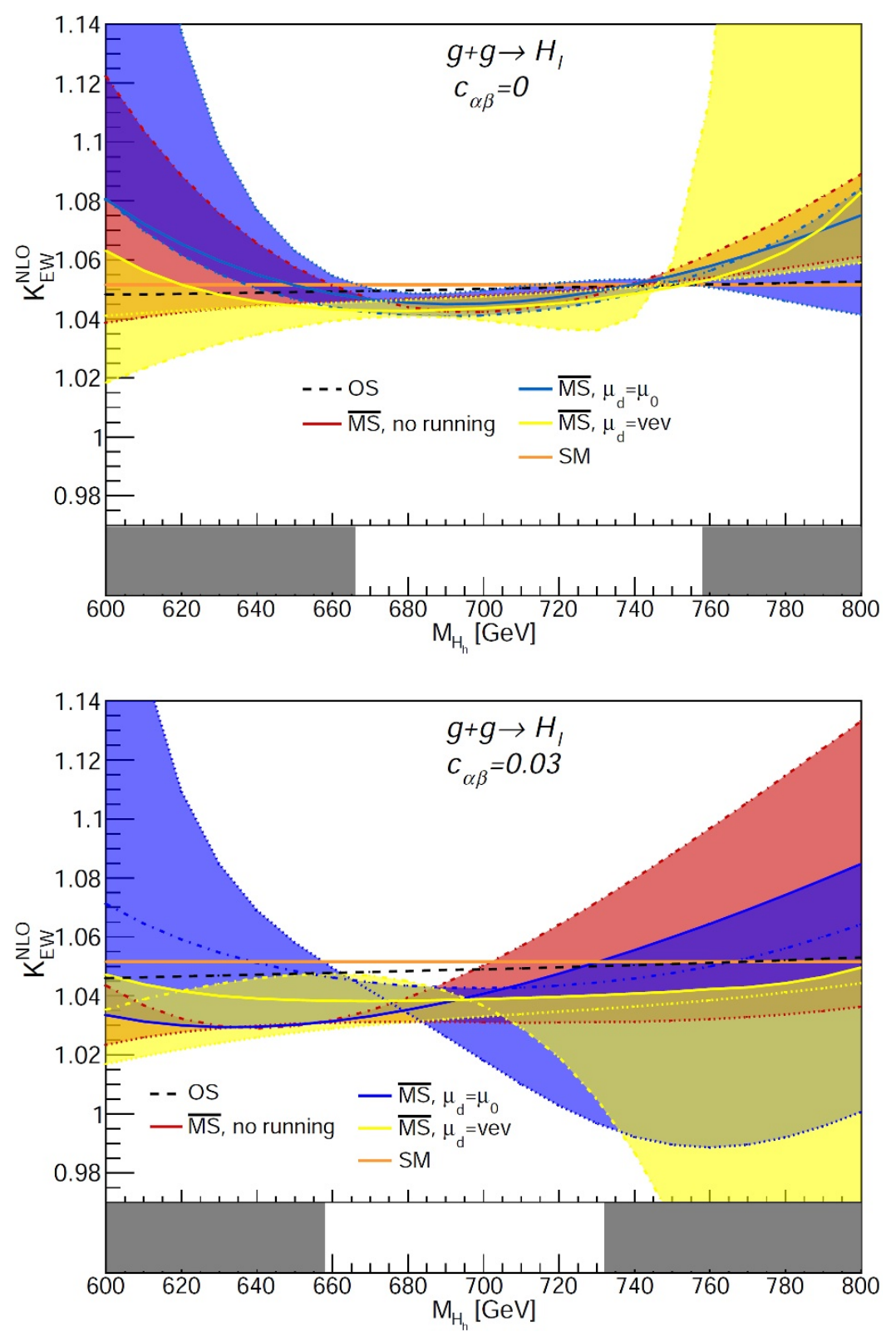

Figure 10. The K-factors of the process $g+g \rightarrow H_{1}$ in various $\overline{\mathrm{MS}}$ schemes are compared to the on-shell scheme and to the SM result. Solid lines are for $\mu=\mu_{0}$, while dotted and dash-dotted lines refer to $\mu=\mu_{0} / 2$ and $\mu=2 \mu_{0}$, respectively. The grey shaded band denotes the region where at least one of the couplings $\left|\lambda_{i}\right| /(4 \pi)(i=1, \ldots 5)$ of the potential in eq. (2.2) becomes larger than 0.5 for at least one of the scales $\mu$ considered. The curve with $\mu=\mu_{0}$ for the case of the $\overline{\mathrm{MS}}$ scheme without running coincides with the $\mu=\mu_{0}$ curve for the $\overline{\mathrm{MS}}$ scheme with running, where the parameters are defined at $\mu_{d}=\mu_{0}$, since in both cases the parameters have been defined at $\mu_{0}$.

renormalization of the mixing angles, the couplings $\lambda_{i}$ of eq. (2.2) are scale dependent. The grey shaded band below each figure shows the region where at least one of the $\overline{\mathrm{MS}}$ couplings $\left|\lambda_{i}\right| /(4 \pi)$ of eq. (2.2) for at least one of the values of $\mu$ becomes larger than 0.5 in the considered renormalization scenarios. Due to the scale dependence, the grey shaded 

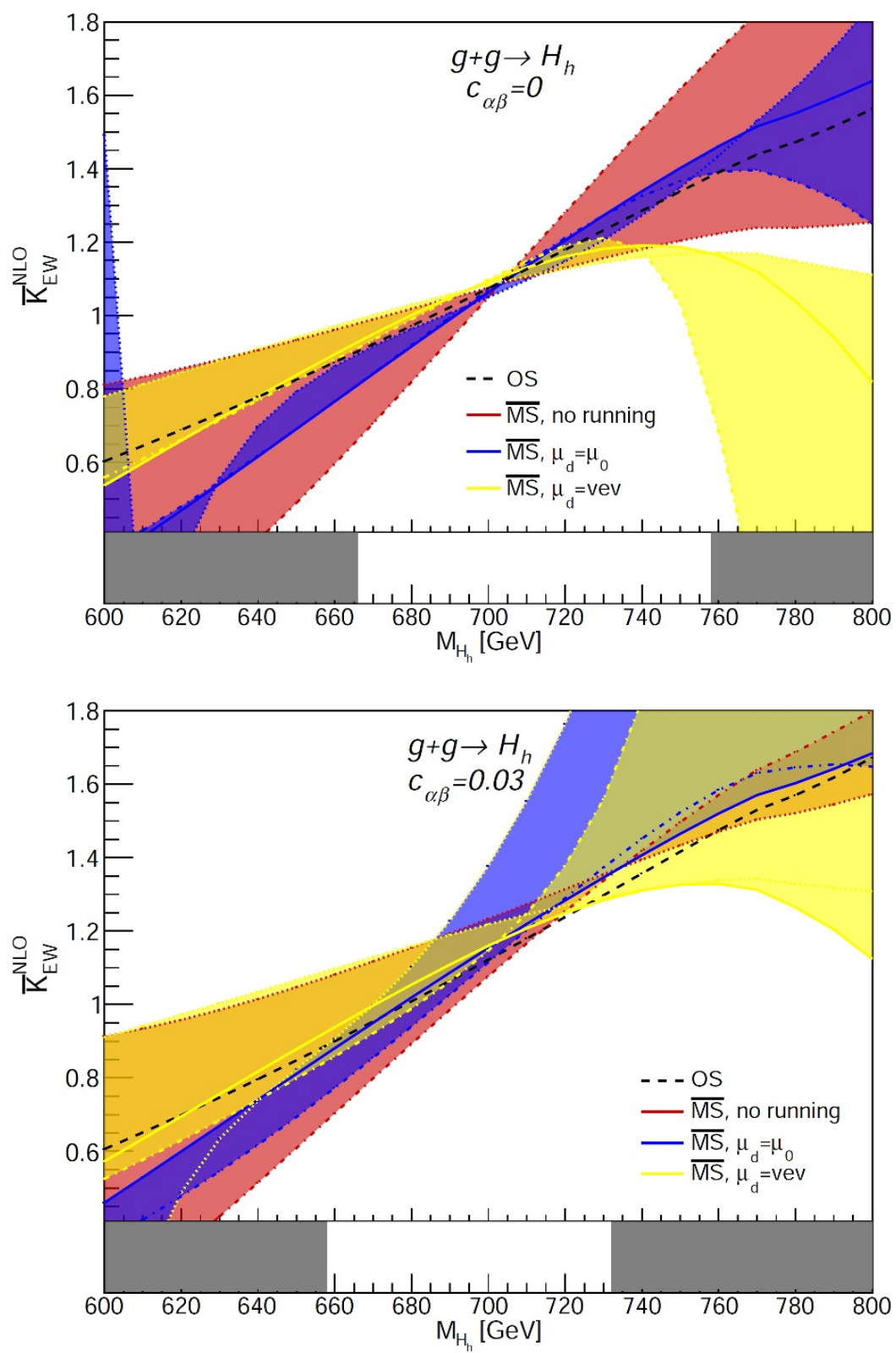

Figure 11. The K-factors of the process $g+g \rightarrow H_{\mathrm{h}}$ in various $\overline{\mathrm{MS}}$ schemes are compared to the on-shell scheme. Solid lines are for $\mu=\mu_{0}$, while dotted and dash-dotted lines refer to $\mu=\mu_{0} / 2$ and $\mu=2 \mu_{0}$, respectively. The grey shaded band denotes the region where at least one of the couplings $\left|\lambda_{i}\right| /(4 \pi)(i=1, \ldots 5)$ of the potential in eq. (2.2) becomes larger than 0.5 for at least one of the scales $\mu$ considered. The curve with $\mu=\mu_{0}$ for the case of the $\overline{\mathrm{MS}}$ scheme without running coincides with the $\mu=\mu_{0}$ curve for the $\overline{\mathrm{MS}}$ scheme with running, where the parameters are defined at $\mu_{d}=\mu_{0}$, since in both cases the parameters have been defined at $\mu_{0}$. 
band for an $\overline{\mathrm{MS}}$ renormalization is in general different from the case of considering a scaleindependent scheme for the mixing angles, as shown in figures 6 and 7. Therefore, the regions where one enters in the non-perturbative regime can also be different.

The K-factor for the process $g+g \rightarrow H_{1}$ is shown in figure 10 for $c_{\alpha \beta}=0$ (upper plot) and $c_{\alpha \beta}=0.03$ (lower plot). In the alignment limit, $c_{\alpha \beta}=0$, the corrections do not differ much from the SM in all considered $\overline{\mathrm{MS}}$ schemes when the couplings $\left|\lambda_{i}\right| /(4 \pi)$ remain below 0.5 (white region); they are just slightly smaller $\left(1.03<K_{\mathrm{EW}}^{\mathrm{NLO}}<1.055\right)$. The situation changes drastically in the non-perturbative grey region, where scale variation becomes large and seems to reveal a certain correlation between perturbativity and scale dependence for this process in the alignment limit. This correlation, however, can be a peculiar property of the chosen scenarios: from eq. (A.1) we see that in the case of a heavy new scalar sector, the largest contribution to the scale dependence in the $\overline{\mathrm{MS}}$ scheme comes from the term proportional to $\left(M_{H_{\mathrm{h}}}^{2}-M_{\mathrm{sb}}^{2}\right)$ when the running of the mixing angles $\alpha$ and $\beta$ as well as of the soft-breaking scale $M_{\mathrm{sb}}$ is not taken into account; the couplings $\lambda_{i}$ of eqs. (B.1)(B.5) on the contrary, show a more complicated dependence on the masses of the neutral sector. In the case of exactly equal heavy masses in the alignment limit, they receive the largest contribution from the $\left(M_{H_{\mathrm{h}}}^{2}-M_{\mathrm{sb}}^{2}\right)$ difference in $\lambda_{1}$. Moving slightly away from the alignment scenario (lower plot with $c_{\alpha \beta}=0.03$ ), we notice a larger difference from the SM value and a wider scale dependence, even in the white (perturbative) region. In particular, for $M_{H_{\mathrm{h}}}$ above $700 \mathrm{GeV}$, even remaining in a region where all $\left|\lambda_{i}\right| /(4 \pi)$ are below 0.5 , the $\overline{\mathrm{MS}}$ bands become quickly wider. Due to this behaviour, $\overline{\mathrm{MS}}$ scale dependence can not serve as a good estimator of the theoretical uncertainty in the light, neutral Higgs-boson production away from the alignment limit.

In figure 11, we compare the different $\overline{\mathrm{MS}}$ schemes for the production of a heavy, neutral Higgs-boson in the alignment limit (upper plot) and slightly away from it (lower plot). In this case we do not have a comparison with the SM and we base our considerations on a comparison with the curve of the on-shell renormalization scheme. Restricting the analysis of the case $c_{\alpha \beta}=0$ to the white (perturbative) region we notice a strong dependence on $M_{H_{\mathrm{h}}}$ for all results. In addition they change a lot for the various $\overline{\mathrm{MS}}$ schemes and for different values of the scale $\mu$. The situation is similar but less dramatic for $c_{\alpha \beta}=0.03$. Owing to these large differences the $\overline{\mathrm{MS}}$ scheme can not reliably predict the NLO contributions to heavy, neutral Higgs-boson production.

Open Access. This article is distributed under the terms of the Creative Commons Attribution License (CC-BY 4.0), which permits any use, distribution and reproduction in any medium, provided the original author(s) and source are credited.

\section{References}

[1] ATLAS collaboration, Observation of a new particle in the search for the Standard Model Higgs boson with the ATLAS detector at the LHC, Phys. Lett. B 716 (2012) 1 [arXiv: 1207.7214] [INSPIRE].

[2] CMS collaboration, Observation of a new boson at a mass of $125 \mathrm{GeV}$ with the CMS experiment at the LHC, Phys. Lett. B 716 (2012) 30 [arXiv:1207.7235] [INSPIRE]. 
[3] Particle Data Group collaboration, C. Patrignani et al., Review of Particle Physics, Chin. Phys. C 40 (2016) 100001 [inSPIRE].

[4] T.D. Lee, A Theory of Spontaneous T Violation, Phys. Rev. D 8 (1973) 1226 [inSPIRE].

[5] R. Barbieri, L.J. Hall and V.S. Rychkov, Improved naturalness with a heavy Higgs: An Alternative road to LHC physics, Phys. Rev. D 74 (2006) 015007 [hep-ph/0603188] [INSPIRE].

[6] Q.-H. Cao, E. Ma and G. Rajasekaran, Observing the Dark Scalar Doublet and its Impact on the Standard-Model Higgs Boson at Colliders, Phys. Rev. D 76 (2007) 095011 [arXiv:0708.2939] [INSPIRE].

[7] ATLAS collaboration, Constraints on new phenomena via Higgs boson couplings and invisible decays with the ATLAS detector, JHEP 11 (2015) 206 [arXiv:1509.00672] [INSPIRE].

[8] ATLAS collaboration, Search for heavy ZZ resonances in the $\ell^{+} \ell^{-} \ell^{+} \ell^{-}$and $\ell^{+} \ell^{-} \nu \bar{\nu}$ final states using proton-proton collisions at $\sqrt{s}=13 \mathrm{TeV}$ with the ATLAS detector, Eur. Phys. J. C 78 (2018) 293 [arXiv:1712.06386] [INSPIRE].

[9] ATLAS collaboration, Search for an additional, heavy Higgs boson in the $H \rightarrow Z Z$ decay channel at $\sqrt{s}=8 \mathrm{TeV}$ in pp collision data with the ATLAS detector, Eur. Phys. J. C 76 (2016) 45 [arXiv: 1507.05930] [INSPIRE].

[10] ATLAS collaboration, Search for Higgs boson pair production in the b $\bar{b} b \bar{b}$ final state from $p p$ collisions at $\sqrt{s}=8 \mathrm{TeV}$ with the ATLAS detector, Eur. Phys. J. C 75 (2015) 412 [arXiv: 1506.00285] [INSPIRE].

[11] ATLAS collaboration, Search for a CP-odd Higgs boson decaying to Zh in pp collisions at $\sqrt{s}=8 \mathrm{TeV}$ with the ATLAS detector, Phys. Lett. B 744 (2015) 163 [arXiv:1502.04478] [INSPIRE].

[12] ATLAS collaboration, Search for a multi-Higgs-boson cascade in $W^{+} W^{-} b \bar{b}$ events with the ATLAS detector in pp collisions at $\sqrt{s}=8$ TeV, Phys. Rev. D 89 (2014) 032002 [arXiv: 1312.1956] [INSPIRE].

[13] C.T. Potter, ATLAS Searches for Beyond the Standard Model Higgs Bosons, in proceedings of the Meeting of the APS Division of Particles and Fields (DPF 2013), Santa Cruz, California, U.S.A., 13-17 August 2013, arXiv: 1310.0515 [INSPIRE].

[14] ATLAS collaboration, Search for Higgs bosons in Two-Higgs-Doublet models in the $H \rightarrow W W \rightarrow e \nu \mu \nu$ channel with the ATLAS detector, ATLAS-CONF-2013-027 [INSPIRE].

[15] CMS collaboration, Combined measurements of the Higgs boson's couplings at $\sqrt{s}=13 \mathrm{TeV}$, CMS-PAS-HIG-17-031 [INSPIRE].

[16] CMS collaboration, Searches for production of two Higgs bosons using the CMS detector, Nucl. Part. Phys. Proc. 273-275 (2016) 764 [InSPIRE].

[17] CMS collaboration, Search for neutral resonances decaying into a $Z$ boson and a pair of $b$ jets or $\tau$ leptons, Phys. Lett. B 759 (2016) 369 [arXiv:1603.02991] [INSPIRE].

[18] CMS collaboration, Search for a low-mass pseudoscalar Higgs boson produced in association with a b $\bar{b}$ pair in pp collisions at $\sqrt{s}=8 \mathrm{TeV}$, Phys. Lett. B 758 (2016) 296 [arXiv: 1511.03610] [INSPIRE].

[19] CMS collaboration, Searches for a heavy scalar boson H decaying to a pair of $125 \mathrm{GeV}$ Higgs bosons hh or for a heavy pseudoscalar boson A decaying to $\mathrm{Zh}$, in the final states with $h \rightarrow \tau \tau$, Phys. Lett. B 755 (2016) 217 [arXiv:1510.01181] [INSPIRE]. 
[20] CMS collaboration, Search for diphoton resonances in the mass range from 150 to $850 \mathrm{GeV}$ in pp collisions at $\sqrt{s}=8 \mathrm{TeV}$, Phys. Lett. B 750 (2015) 494 [arXiv:1506.02301] [INSPIRE].

[21] CMS collaboration, Search for a pseudoscalar boson decaying into a $Z$ boson and the 125 GeV Higgs boson in $\ell^{+} \ell^{-} b \bar{b}$ final states, Phys. Lett. B 748 (2015) 221 [arXiv: 1504.04710] [INSPIRE].

[22] LHC Higgs Cross Section Working Group, D. de Florian et al., Handbook of LHC Higgs Cross Sections: 4. Deciphering the Nature of the Higgs Sector, arXiv:1610.07922 [INSPIRE].

[23] H.M. Georgi, S.L. Glashow, M.E. Machacek and D.V. Nanopoulos, Higgs Bosons from Two Gluon Annihilation in Proton Proton Collisions, Phys. Rev. Lett. 40 (1978) 692 [InSPIRE].

[24] G. Passarino, C. Sturm and S. Uccirati, Complete Electroweak Corrections to Higgs production in a Standard Model with four generations at the LHC, Phys. Lett. B 706 (2011) 195 [arXiv: 1108.2025] [INSPIRE].

[25] A. Denner et al., Higgs Production and Decay with a Fourth Standard-Model-Like Fermion Generation, Eur. Phys. J. C 72 (2012) 1992 [arXiv:1111.6395] [InSPIRE].

[26] A. Denner, L. Jenniches, J.-N. Lang and C. Sturm, Gauge-independent $\overline{M S}$ renormalization in the 2HDM, JHEP 09 (2016) 115 [arXiv: 1607.07352] [INSPIRE].

[27] A. Denner, J.-N. Lang and S. Uccirati, NLO electroweak corrections in extended Higgs Sectors with RECOLA2, JHEP 07 (2017) 087 [arXiv:1705.06053] [INSPIRE].

[28] L. Altenkamp, S. Dittmaier and H. Rzehak, Renormalization schemes for the Two-Higgs-Doublet Model and applications to $h \rightarrow W W / Z Z \rightarrow 4$ fermions, JHEP 09 (2017) 134 [arXiv: 1704.02645] [INSPIRE].

[29] L. Altenkamp, S. Dittmaier and H. Rzehak, Precision calculations for $h \rightarrow W W / Z Z \rightarrow 4$ fermions in the Two-Higgs-Doublet Model with Prophecy4f, JHEP 03 (2018) 110 [arXiv: 1710.07598] [INSPIRE].

[30] S. Actis, G. Passarino, C. Sturm and S. Uccirati, NLO Electroweak Corrections to Higgs Boson Production at Hadron Colliders, Phys. Lett. B 670 (2008) 12 [arXiv:0809.1301] [INSPIRE].

[31] S. Actis, G. Passarino, C. Sturm and S. Uccirati, NNLO Computational Techniques: The Cases $H \rightarrow \gamma \gamma$ and $H \rightarrow g g$, Nucl. Phys. B 811 (2009) 182 [arXiv:0809.3667] [InSPIRE].

[32] S. Kanemura, T. Kasai and Y. Okada, Mass bounds of the lightest CP even Higgs boson in the two Higgs doublet model, Phys. Lett. B 471 (1999) 182 [hep-ph/9903289] [InSPIRE].

[33] G.C. Branco, L. Lavoura and J.P. Silva, CP Violation, Int. Ser. Monogr. Phys. 103 (1999) 1 [INSPIRE].

[34] S.L. Glashow and S. Weinberg, Natural Conservation Laws for Neutral Currents, Phys. Rev. D 15 (1977) 1958 [INSPIRE].

[35] E.A. Paschos, Diagonal Neutral Currents, Phys. Rev. D 15 (1977) 1966 [InSPIRE].

[36] J.F. Gunion and H.E. Haber, The CP conserving two Higgs doublet model: The Approach to the decoupling limit, Phys. Rev. D 67 (2003) 075019 [hep-ph/0207010] [INSPIRE].

[37] J. Fleischer and F. Jegerlehner, Radiative Corrections to Higgs Decays in the Extended Weinberg-Salam Model, Phys. Rev. D 23 (1981) 2001 [InSPIRE].

[38] M. Krause, R. Lorenz, M. Mühlleitner, R. Santos and H. Ziesche, Gauge-independent Renormalization of the 2-Higgs-Doublet Model, JHEP 09 (2016) 143 [arXiv:1605.04853] [INSPIRE]. 
[39] S. Actis, A. Ferroglia, M. Passera and G. Passarino, Two-Loop Renormalization in the Standard Model. Part I: Prolegomena, Nucl. Phys. B 777 (2007) 1 [hep-ph/0612122] [INSPIRE].

[40] M. Krause, M. Mühlleitner, R. Santos and H. Ziesche, Higgs-to-Higgs boson decays in a 2HDM at next-to-leading order, Phys. Rev. D 95 (2017) 075019 [arXiv:1609.04185] [INSPIRE].

[41] S. Kanemura, M. Kikuchi and K. Yagyu, Fingerprinting the extended Higgs sector using one-loop corrected Higgs boson couplings and future precision measurements, Nucl. Phys. B 896 (2015) 80 [arXiv: 1502.07716] [INSPIRE].

[42] J.R. Espinosa and I. Navarro, Scale independent mixing angles, Phys. Rev. D 66 (2002) 016004 [hep-ph/0109126] [INSPIRE].

[43] J.R. Espinosa and Y. Yamada, Scale independent and gauge independent mixing angles for scalar particles, Phys. Rev. D 67 (2003) 036003 [hep-ph/0207351] [INSPIRE].

[44] J.M. Cornwall, Dynamical Mass Generation in Continuum QCD, Phys. Rev. D 26 (1982) 1453 [INSPIRE].

[45] J.M. Cornwall and J. Papavassiliou, Gauge Invariant Three Gluon Vertex in QCD, Phys. Rev. D 40 (1989) 3474 [INSPIRE].

[46] J. Papavassiliou, Gauge Invariant Proper Selfenergies and Vertices in Gauge Theories with Broken Symmetry, Phys. Rev. D 41 (1990) 3179 [inSPIRE].

[47] G. Degrassi and A. Sirlin, Gauge invariant selfenergies and vertex parts of the Standard Model in the pinch technique framework, Phys. Rev. D 46 (1992) 3104 [INSPIRE].

[48] G. Degrassi, B.A. Kniehl and A. Sirlin, Gauge-invariant formulation of the $S$, T, and $U$ parameters, Phys. Rev. D 48 (1993) R3963 [InSPIRE].

[49] J. Papavassiliou, Gauge independent transverse and longitudinal self energies and vertices via the pinch technique, Phys. Rev. D 50 (1994) 5958 [hep-ph/9406258] [INSPIRE].

[50] J. Papavassiliou and A. Pilaftsis, Gauge invariance and unstable particles, Phys. Rev. Lett. 75 (1995) 3060 [hep-ph/9506417] [INSPIRE].

[51] J. Papavassiliou and A. Pilaftsis, A Gauge independent approach to resonant transition amplitudes, Phys. Rev. D 53 (1996) 2128 [hep-ph/9507246] [INSPIRE].

[52] J. Papavassiliou and A. Pilaftsis, Gauge invariant resummation formalism for two point correlation functions, Phys. Rev. D 54 (1996) 5315 [hep-ph/9605385] [INSPIRE].

[53] B.A. Kniehl, C.P. Palisoc and A. Sirlin, Higgs boson production and decay close to thresholds, Nucl. Phys. B 591 (2000) 296 [hep-ph/0007002] [InSPIRE].

[54] D. Binosi and J. Papavassiliou, Pinch technique and the Batalin-Vilkovisky formalism, Phys. Rev. D 66 (2002) 025024 [hep-ph/0204128] [INSPIRE].

[55] D. Binosi and J. Papavassiliou, The Two loop pinch technique in the electroweak sector, Phys. Rev. D 66 (2002) 076010 [hep-ph/0204308] [INSPIRE].

[56] G. 't Hooft and M.J.G. Veltman, Scalar One Loop Integrals, Nucl. Phys. B 153 (1979) 365 [INSPIRE].

[57] G. Passarino and M.J.G. Veltman, One Loop Corrections for $e^{+} e^{-}$Annihilation Into $\mu^{+} \mu^{-}$ in the Weinberg Model, Nucl. Phys. B 160 (1979) 151 [INSPIRE]. 
[58] A. Alloul, N.D. Christensen, C. Degrande, C. Duhr and B. Fuks, FeynRules 2.0 - A complete toolbox for tree-level phenomenology, Comput. Phys. Commun. 185 (2014) 2250 [arXiv: 1310.1921] [INSPIRE].

[59] S. Actis, A. Ferroglia, G. Passarino, M. Passera, C. Sturm and S. Uccirati, GraphShot, a Form package for automatic generation and manipulation of one- and two-loop Feynman diagrams, unpublished.

[60] P. Nogueira, Automatic Feynman graph generation, J. Comput. Phys. 105 (1993) 279 [INSPIRE].

[61] J.A.M. Vermaseren, New features of FORM, math-ph/0010025 [INSPIRE].

[62] J. Kuipers, T. Ueda, J.A.M. Vermaseren and J. Vollinga, FORM version 4.0, Comput. Phys. Commun. 184 (2013) 1453 [arXiv:1203.6543] [INSPIRE].

[63] G. Passarino, An Approach toward the numerical evaluation of multiloop Feynman diagrams, Nucl. Phys. B 619 (2001) 257 [hep-ph/0108252] [INSPIRE].

[64] G. Passarino and S. Uccirati, Algebraic numerical evaluation of Feynman diagrams: Two loop selfenergies, Nucl. Phys. B 629 (2002) 97 [hep-ph/0112004] [InSPIRE].

[65] A. Ferroglia, M. Passera, G. Passarino and S. Uccirati, Two loop vertices in quantum field theory: Infrared convergent scalar configurations, Nucl. Phys. B 680 (2004) 199 [hep-ph/0311186] [INSPIRE].

[66] G. Passarino and S. Uccirati, Two-loop vertices in quantum field theory: Infrared and collinear divergent configurations, Nucl. Phys. B 747 (2006) 113 [hep-ph/0603121] [INSPIRE].

[67] S. Actis, A. Ferroglia, G. Passarino, M. Passera and S. Uccirati, Two-loop tensor integrals in quantum field theory, Nucl. Phys. B 703 (2004) 3 [hep-ph/0402132] [InSPIRE].

[68] J. Baglio, O. Eberhardt, U. Nierste and M. Wiebusch, Benchmarks for Higgs Pair Production and Heavy Higgs boson Searches in the Two-Higgs-Doublet Model of Type II, Phys. Rev. D 90 (2014) 015008 [arXiv: 1403.1264] [INSPIRE].

[69] D. Chowdhury and O. Eberhardt, Update of Global Two-Higgs-Doublet Model Fits, JHEP 05 (2018) 161 [arXiv: 1711.02095] [INSPIRE].

[70] P. Basler, P.M. Ferreira, M. Mühlleitner and R. Santos, High scale impact in alignment and decoupling in two-Higgs doublet models, Phys. Rev. D 97 (2018) 095024 [arXiv:1710.10410] [INSPIRE].

[71] A. Denner, S. Dittmaier, M. Roth and D. Wackeroth, Predictions for all processes $e^{+} e^{-} \rightarrow$ fermions $+\gamma$, Nucl. Phys. B 560 (1999) 33 [hep-ph/9904472] [INSPIRE].

[72] A. Denner, S. Dittmaier, M. Roth and L.H. Wieders, Electroweak corrections to charged-current $e^{+} e^{-} \rightarrow 4$ fermion processes: Technical details and further results, Nucl. Phys. B 724 (2005) 247 [Erratum ibid. B 854 (2012) 504] [hep-ph/0505042] [INSPIRE].

[73] A. Denner and S. Dittmaier, The Complex-mass scheme for perturbative calculations with unstable particles, Nucl. Phys. Proc. Suppl. 160 (2006) 22 [hep-ph/0605312] [InSPIRE].

[74] J.A.M. Vermaseren, Axodraw, Comput. Phys. Commun. 83 (1994) 45 [inSPIRE].

[75] J.C. Collins and J.A.M. Vermaseren, Axodraw Version 2, arXiv: 1606.01177 [InSPIRE]. 